\title{
Ferroelectret materials and devices for energy harvesting applications
}

\author{
Yan Zhanga, b, Chris Rhys Bowena, Sujoy Kumar Ghosh, Dipankar Mandalc, \\ Hamideh Khanbareha, Mustafa Arafad ${ }^{d}$ Chaoying Wan ${ }^{\mathrm{e}}$
}

\footnotetext{
${ }^{a}$ Materials and Structures Centre, Mechanical Engineering, University of Bath, Bath, BA2 7AY, UK

${ }^{b}$ State Key Laboratory of Powder Metallurgy, Central South University, Changsha, 410083, China

${ }^{c}$ Department of Physics, Jadavpur University, Kolkata, India

${ }^{d}$ Mechanical Engineering Department, American University in Cairo, Egypt

e International Institute for Nanocomposites Manufacturing, University of Warwick, CV4 7AL, UK
}

\begin{abstract}
This paper provides an overview of ferroelectret materials for energy harvesting applications. These materials take the form of a cellular compliant polymer with polarised pores that provide a piezoelectric response to generate electrical energy as a result of an applied strain or surrounding vibration. The manufacturing processes used to create ferroelectret polymer structures for energy harvesting are discussed, along with the range of microstructural features and pore sizes that are formed. Their important mechanical, electrical and harvesting performance are then described and compared. Modelling approaches for microstructural design or for predicting the vibrational and frequency dependent response are examined. Finally, conclusions and future perspectives for ferroelectret materials for energy harvesting applications are provided.
\end{abstract}




\section{Introduction}

Porous non-polar polymers that exhibit ferroelectric-like behaviour when subjected to a high electric field can be classified as ferroelectret materials [1]. Ferroelectrets are a class of piezoelectrically-active polymer foam whereby a gas, such as air, within a macro-sized pore space (typically $>1 \mu \mathrm{m}$ ) can be subject to electrical breakdown during the application of a high electric field by a corona poling process. The microplasma discharges which are formed as a consequence of the breakdown of air within the pore space are deposited on the upper and lower pore surfaces of the polymer [2], as shown in Fig. 1(A). This structure has some similarities to the atomic scale dipole configuration in ferroelectric materials, which originates from the asymmetric arrangement of the negative and positive atoms and remnant polarisation after being subjected to an electric field above its coercive field during a similar poling process. As with ferroelectrics, ferroelectret materials can also exhibit dipole switching and polarity reversal as the direction of the applied electric field is switched, leading to the formation of a theoretical hysteresis loop as shown in Fig. 1(B). In such a loop the threshold voltage in Fig. 1(B) for breakdown within a pore is analogous to the coercive field for a ferroelectric. Once the polarised pores are formed, an electroded ferroelectret will attract surface charges, as in Fig. $1 \mathrm{~A}$, and if the polarisation of the pores is changed by a mechanical stress or a temperature change there is a redistribution of surface charge and an electric current will flow. Ferroelectrets are therefore both piezoelectric and pyroelectric and such properties have recently attracted interest in energy harvesting applications. Ferroelectrets are also referred to as piezoelectrets or piezo(ferro)electrets [3-6]; although we will describe these polarised cellular materials as ferroelectrets throughout the review for consistency and ease of any analogies with ferroelectrics ${ }^{1}$.

Energy harvesting involves scavenging ambient sources of energy, which are often otherwise wasted and converting them into usable electrical energy; examples of

\footnotetext{
${ }^{1}$ For example, not all piezoelectrics are ferroelectric and exhibit polarization reversal with an applied electric field (e.g. quartz, AIN, ZnO).
} 
energy sources include heat, light and mechanical vibrations. This is often achieved with the help of electroactive materials or structures [7-10] and the advantage of energy harvesting is that a wired external electrical power source or battery is not necessary, resulting in more compact device configuration, less maintenance and reduced cost. While conventional ferroelectrics have attracted interest in both piezoelectric (vibration) and pyroelectric harvesting (heat fluctuations), ferroelectrets have been predominately considered to date for harvesting mechanical motion. This is a result of their high piezoelectric coefficients; for example piezoelectric coefficients (charge per unit force) in the poling direction as high as $d_{33} \sim 495 \mathrm{pC} / \mathrm{N}$ have been achieved in a ferroelectret polymer [11], compared with $d_{33} \sim-17 \mathrm{pC} / \mathrm{N}$ for a polyvinylidene difluoride (PVDF) ferroelectric polymer [12] and $d_{33} \sim 500 \mathrm{pC} / \mathrm{N}$ in a lead zirconate titanate (PZT) ferroelectric ceramic which is brittle (low fracture toughness) and has a high stiffness compared to the porous polymer ferroelectret materials [13].

As described by Uchino [14] and Deutz et al. [15], $d_{33}$ is not the only factor for predicting energy harvesting performance, and for piezoelectric materials the energy harvesting figure of merit $\left(\mathrm{FoM}_{33}\right)$ has been derived by Islam and Priya [16] and this is of relevance for ferroelectrets where:

$$
F_{0 M}=d_{33}^{2} / \varepsilon_{33}^{X}
$$

where $\varepsilon_{33}^{X}$ is the permittivity at constant stress. Clearly the advantage of ferroelectrets is the combination of a high piezoelectric $d_{33}$ coefficient and a low permittivity of the cellular polymer structure.

Less work has considered ferroelectrets for pyroelectric harvesting since they simply exhibit much smaller pyroelectric coefficients, e.g. $\sim 0.25 \mu \mathrm{C} / \mathrm{cm}^{2} \mathrm{~K}$ in ferroelectret polypropylene [17] compared to $-27 \mu \mathrm{C} / \mathrm{cm}^{2} \mathrm{~K}$ for ferroelectric PVDF [18] and 450 $\mu \mathrm{C} / \mathrm{cm}^{2} \mathrm{~K}$ for a PZT [19]. The poor pyroelectric coefficients of a ferroelectret, compared to its excellent piezoelectric response are a result of the highly compliant nature of ferroelectrets, which leads to a large change in dimension of the charged pore with pressure and therefore large changes in polarisaton under mechanical loads; however there is likely to be only a small change is pore size with temperature. In contrast, for 
a ferroelectric the polarisation is at the atomic scale and changes to a much larger degree with temperature, leading to high pyroelectric coefficients. While the temperature insensitive characteristic of ferroelectrets makes them poor thermal sensors or harvesters, it has the potential to be beneficial for thermally stable electromechanical devices, such as mechanical sensors, actuators, keyboards, microphones and loudspeakers.

The aim of this review is to provide an overview of ferroelectret materials for energy harvesting applications; in particular the harvesting of vibration and strain, which leads to changes in the dimensions of the polarised pores, and a change in the level of bound charge to produce an electric current. A number of other excellent reviews and book chapters exist with regard to ferroelectret materials $[20,21]$ which mainly focus on their manufacture routes $[18,22]$, fundamental properties $[23,24]$, modelling methods [25], and applications related to transducers such as sensors $[1,2,26]$. Electrets that exhibit no hysteresis behaviour are also of interest but are not covered in this review and the reader is refereed to some key summaries in this area $[27,28]$.

This review will provide an overview of the materials and manufacturing methods used for ferroelectrets, with a particular emphasis on those used for energy harvesting devices and nanogenerator applications. The characterisation and properties are then described, along with modelling of the materials and, in particular, their response to dynamic mechanical loads since the materials are likely to be used in vibration energy harvesting applications. Finally, examples of the applications of ferroelectrets in energy harvesting are overviewed along with potential future avenues of research. 
(B)
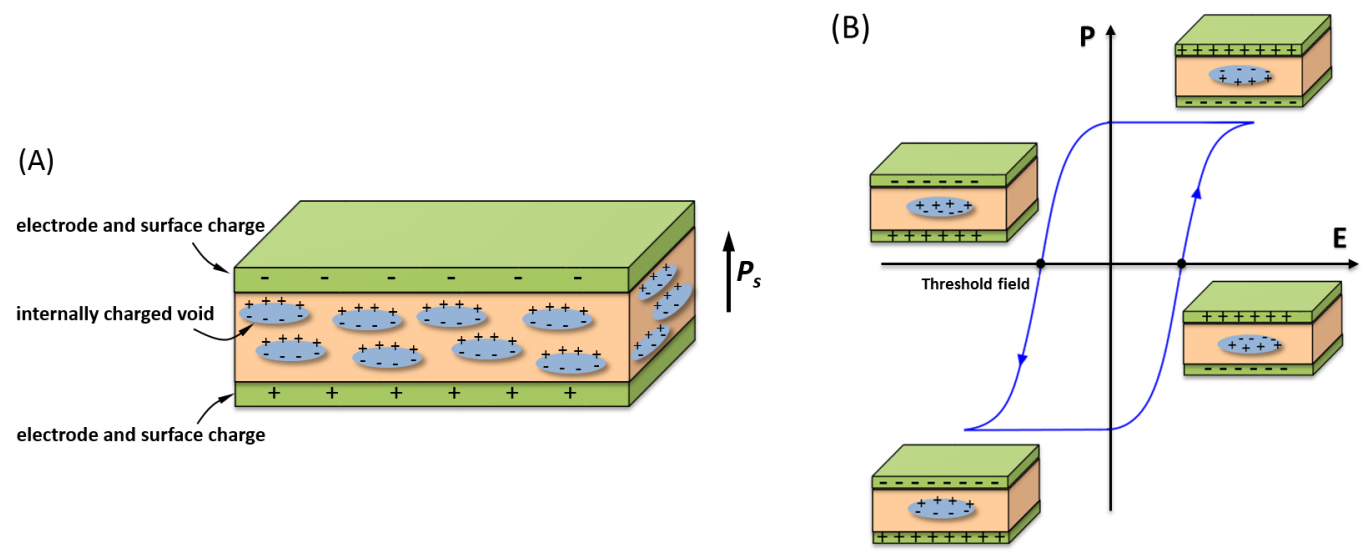

Fig. 1 (A) Schematic of ferroelectret material where $P_{s}$ is the polarisation direction and $(B)$ the hysteresis loop in a single-pore ferroelectret.

\section{Materials and manufacture of cellular ferroelectrets and nanogenerators}

\subsection{Fundamental Mechanism}

Fig. 2 illustrates the working mechanism of the ferroelectrets. The pores whose surface must be smooth and continuous without holes [29] can be charged as a result of Townsend breakdown induced by the application of high poling field, e.g. direct contact poling or corona poling (Fig. 2(A)). Trapped charges with opposite polarities are then formed on the upper and lower internal surfaces of the voids, to create a dipole-like macroscopic structure, as shown in Fig. 2(B). Unlike the ion displacements in a unit cell lattice of traditional ferroelectric materials when subjected to mechanical stress, the macroscopic electromechanical behaviour of ferroelectrets are a result of the deformation of charged voids, leading to the change of the polarisation. Accordingly, the bound surface charges are free to flow in an electric circuit, shown in Fig. 2(C), which is the basic macroscopic mechanism for ferroelectrets used for, sensing [30, 31], and energy harvesting [32, 33]applications. For actuation, the application of an electric field leads to elongation of the pores, and the reader is referred to $[34,35]$ for further details in this area. 
The longer the trapped charges can be sustained at the pores, the more stable the dipole-like macroscopic structure becomes, leading to better piezoelectric performance of the ferroelectret with time. There are a number of factors influencing the stabilisation of the trapped charges at/near the internal interfaces, these include the nature of the polymer and its electrical properties such conductivity and dielectric loss, the ratio between the width and length of the elongated pore channel, the thickness of the sample, and any surface modification. This fundamental behaviour of ferroelectret has been reported broadly in a number of excellent general reviews and books $[1,22,23,36,37]$.

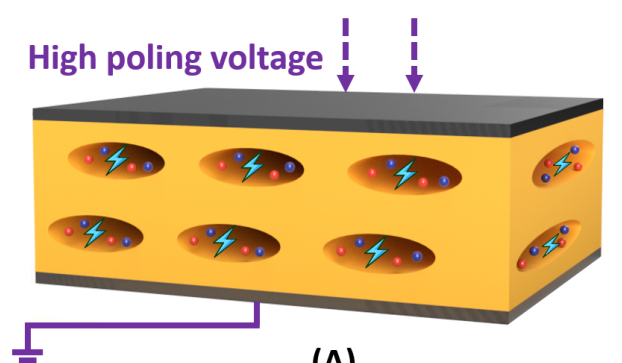

(A)

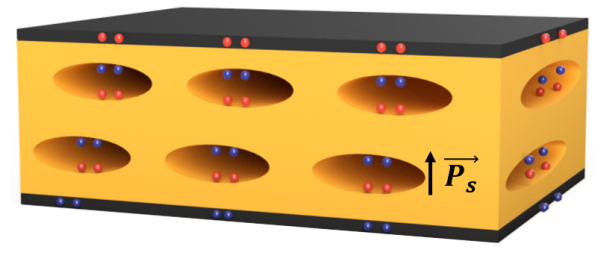

(B)

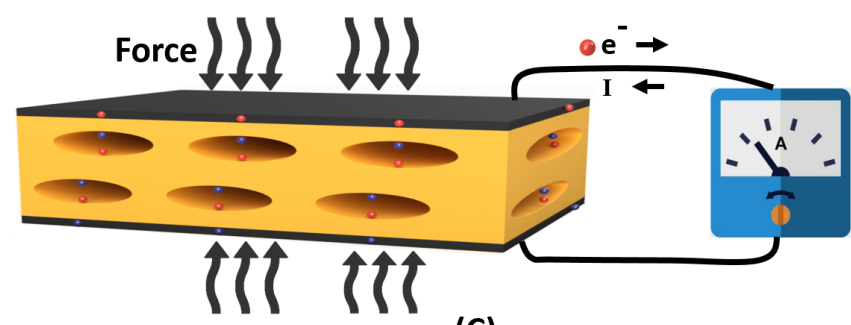

(C)

\& Townsend breakdown $\odot$ Negative charge

Positive charge Electrode

Fig. 2 Schematic of the working mechanism of ferroelectret. (a) Townsend breakdown under a high poling voltage, that follows Paschen's law for breakdown in air, (b) charged sample having positive/negative electrical charges on the opposite faces of the polymer surfaces with the polarisation of $\overrightarrow{P_{S}}$, (c) piezoelectrically induced charge flow when external force applied along the polarisation direction. 


\subsection{Materials}

While conventional ferroelectric polymers, such as polyvinylidene difluoride (PVDF) and its copolymers [38], generate piezoelectricity due to the ferroelectric orientation of dipolar crystals, cellular ferroelectret polymers have expanded the applications of non-polar polymers as high performance piezoelectric materials. Thermoplastic and non-polar polymers such as polyurethane (PU), polyolefin (PO), cyclo-olefin polymers (COP) have been used to create ferroelectrets. Copolymers (COC), polyvinyl chloride (PVC), fluoropolymer, poly (ethylene terephthalate) (PET) and poly (ethylene napthalate) (PEN) have also been used, and the range of polymers used for energy harvesting applications is outlined in Table 1 . Since there is a need to maintain the highly charged pores, the polymer should ideally be a good dielectric and electrically insulating. The polymer ferroelectret foam can be considered as a composite consisting of a gas phase dispersed in a solid polymer matrix, where the gases are generated using physical or chemical blowing agents, which dissolve in liquid polymer or polymer melt under high temperature and pressure.

\subsection{Manufacturing}

During the poling process, as the applied electric field in the pore space reaches a threshold electric field, as in Fig. 1B, there is initiation of Townsend breakdown [39] for dielectric barrier discharge. The required voltage is based on Paschen's law [40] and the charged pores create the dipole structure of the ferroelectrets. Either contact poling or corona poling are effective to achieve a polarized porous polymer when the electric field reaches the critical breakdown field [41] to initiate air breakdown. To obtain a high electro-mechanical response, a cellular geometry or lens-shaped pore is desirable as shown in Fig. $3 \mathrm{~A}$ due to the relatively low elastic stiffness of such a configuration in the polarisation direction [40], with the width and length of the void typically $>10 \mu \mathrm{m}$ and few $\mu \mathrm{m}[42,43]$. The variation of breakdown voltage and field with pore height (spacing) is shown in Fig. $3 \mathrm{~B}$, where at a pressure of 1 atmosphere the $y$-axis corresponds to spacing. 

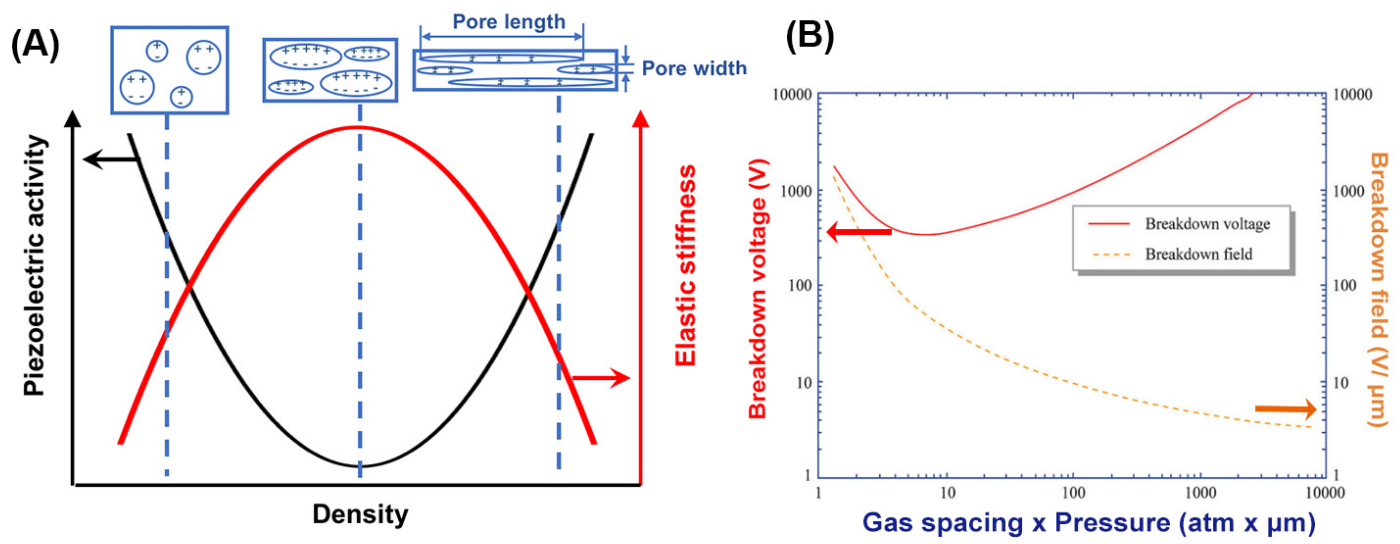

Fig. 3 (A) Schematic of the relationship between piezoelectric activity (thick black line), elastic stiffness (thin red line) and sample density [40] (Reproduced with permission, Copyright 2006, John Wiley and Sons), (B) influence of gap spacing between top and bottom electrodes on the gaseous breakdown voltage at a pressure of 1 atmosphere [44]. Creative commons licence.

\subsubsection{Foaming processes}

Various foaming technologies, such as injection moulding and extrusion foaming, and cross-linked polyolefin foaming have been applied to create polymer based cellular structures for ferroelectrets. Alternatively, cellular structures are also created by stretching particulate filled polymers, where the voids originate from the interfacial delamination between different materials. As outlined in Fig. 2, a poling treatment of the cellular structure is necessary through direct electrode contact, corona or soft Xray poling that leads to Townsend breakdown of the gas inside the voids, thus creating macro-dipoles. Therefore, thermoplastic closed celled foam structures are useful for ferroelectrets.

The dimension, shape and porosity of polymer foams affect the piezoelectric response. Polymeric ferroelectrets are generally macrocellular structures, with cell sizes of $>50 \mu \mathrm{m}$ and cell densities $<10^{6}$ cells $/ \mathrm{cm}^{3}$ [45]. Small pore sizes $(<<10 \mu \mathrm{m})$ or very large pores (>>100 $\mu \mathrm{m}$ ) are undesirable because they require large voltages for breakdown, see Fig. 3B. The size of the voids can be varied by the foaming conditions such as polymer viscosity, composition, foaming pressure and temperature [46, 47], inflation conditions, as well as post-processing parameters (time, temperature, and 
pressure). The shape of the voids, in particular the aspect ratio, can be adjusted by stretching or compression. Anisotropic foams with flat disk or lens-like closed-cell voids with an aspect ratio (ratio of cell length/height) greater than four are preferable for ferroelectrets; this provides a sufficiently low compliance to ensure a large change in pore shape and therefore polarisation with an applied load, see Figure 3A. [24, 26, 48]

Polyethylene-naphthalate (PEN) ferroelectrets with average pore heights below $8 \mu \mathrm{m}$ were prepared by using a 'voiding - inflation - stretching' process. The piezoelectric coefficient $d_{33}$ was increased by increasing the poling voltage from $4 \mathrm{kV}$ to $8 \mathrm{kV}$. A higher $d_{33}$ was observed when the samples were charged at elevated temperature just below the glass transition temperature of PEN, although the elastic modulus of the PEN ferroelectrets was decreased upon thermal treatment [49]. When the polypropylene (PP)-foamed films were exposed to a gradual temperature and pressure increase, an appropriate cellular structure with high aspect ratio $\sim 6.6$ was obtained. This structure led to a high quasi-static piezoelectric $d_{33}$ coefficient of 800 $\mathrm{pC} / \mathrm{N}$ (45\% higher than untreated ones) [50]. Wu et al. [51] used a biaxial orientated polypropylene film, as seen in Fig. 4, which was initially placed in a chamber at $2 \mathrm{MPa}$ at $100^{\circ} \mathrm{C}$ for three hours. A rapid decrease of pressure leads to the film expanding forming oval shaped pores for subsequent charging by a poling process and electroding to create the final device for self-powered health sensors.

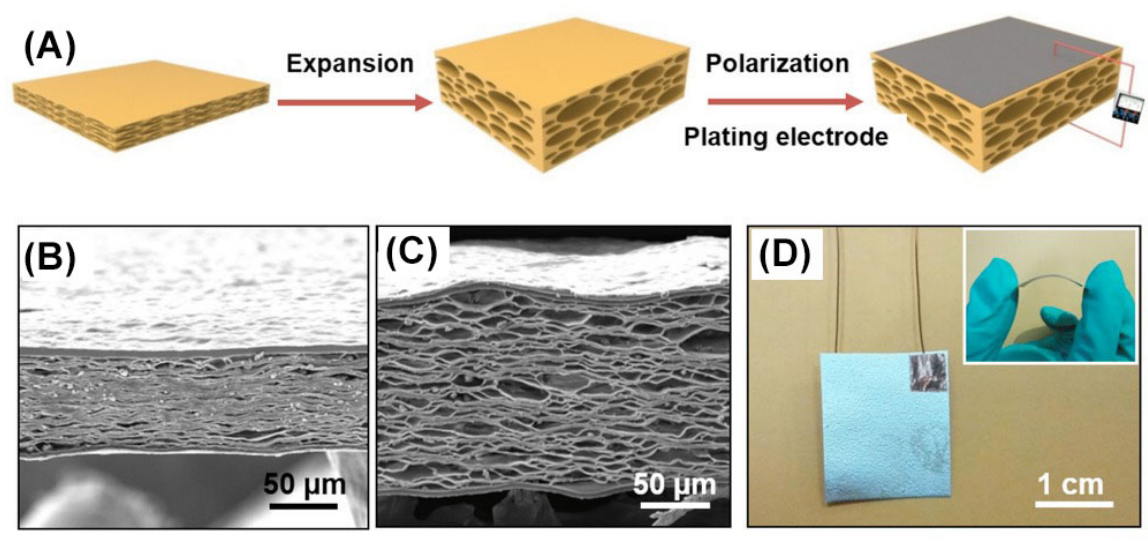

Fig. 4 (A) Schematic of fabrication process. Cross-section view of cellular polypropylene (B) before and (C) after expansion process. (D) Image of final poled and electroded device [51]. (Reproduced with permission, Copyright 2015, John Wiley and Sons) 
A green foaming process has been developed that uses a supercritical fluid, such as carbon dioxide instead of chemical blowing agents [52]. The metastable gas dispersion is then locked in the polymer matrix via solidification during the foam expansion process. The solidification process can be completed via crosslinking of the polymeric precursors, or vitrification or crystallization of the polymer matrices.

Luo et al. [53] used a blowing agent that was mixed with a polymer solution to create thick film ferroelectrets for energy harvesting that could be moulded and cured into the desired shape. The ferroelectrets could be formed with a thickness of several hundred microns to several millimetres and the void size was controlled by heating time and temperature. As a newly developed alternative to foaming, Yan et al. recently used freeze casting as a route to form aligned porous polymeric microstructures [54].

\subsubsection{Sandwich layer architectures}

Porous sandwich structures consisting of dense outer layers of fluorinated ethylene propylene (FEP) and an inner porous layer based on a fibrous polytetrafluoroethylene (f-PTFE) have been formed for self-powered sensors; see Fig. 5. These were produced by a low-cost hot pressing at $5 \mathrm{MPa}$ and $\sim 285^{\circ} \mathrm{C}$, followed by metallisation and corona poling. The additional interfaces between the FEP and f-PTFE was thought to improve surface charge density, Fig. 5e [55], and resulting electromechanical properties. An innovative and simple approach to prepare sandwich structure was presented by Shi et al. [5] who used a conventional porous foam sandwiched between two FEP films with aluminium upper and lower electrodes. 

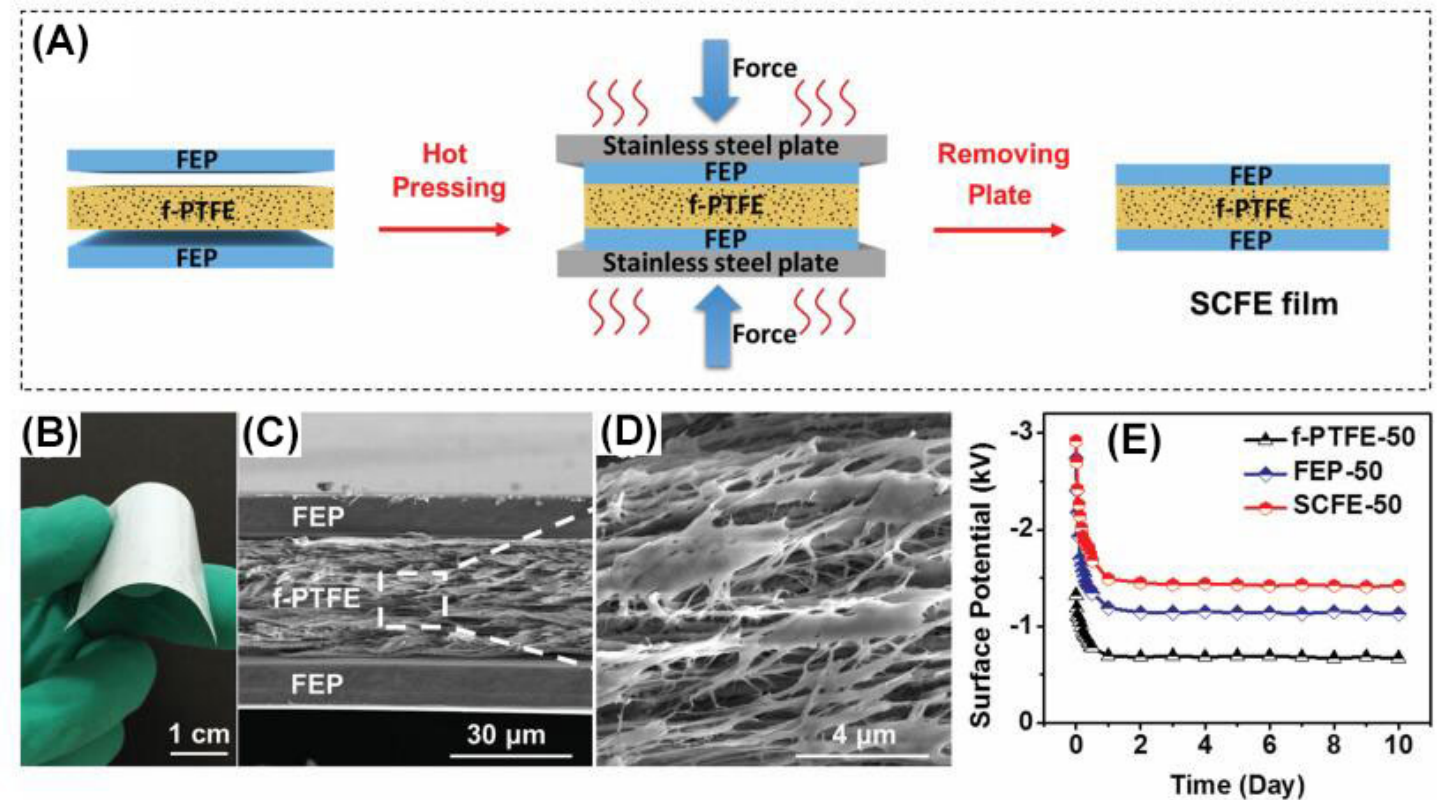

Fig. 5 Fabrication of sandwich composite fluorocarbon (SCF) film. a) Schematic of fabrication process.

b) Image of film, and c) cross-section of SCF film. d) Enlarged view of f-PTFE layer in the SCF film. e) Surface potential decay with time of f-PTFE, FEP, and SCF films of same thickness of 50 $\mu \mathrm{m}$. [55]

(Reprinted with permission John Wiley and Sons.)

\subsubsection{Micro-patterning methods}

Pores have also been produced by a variety of micro-patterning processes to create ferroelectrets for energy harvesting. Zhang et al. $[3,4,6]$ used a process outlined in Fig. 6 whereby FEP was used as the material which was fused together at high temperature. A soft pad with two thin FEP films on its upper and lower surfaces was clamped between two patterned templates with a grooved architecture (see Fig. 6B). Hot pressing at $100^{\circ} \mathrm{C}$ leads to patterning of the films as the FEP fills the grooved structure (Fig. 6C). The soft interlayer pad is subsequently removed (Fig. 6D) and the two FEP films are then fusion bonded at a higher temperature of $320^{\circ} \mathrm{C}$ (Fig. $6 \mathrm{E}$ ) to create the FEP porous structure (Fig. 6F).

Shi et al. [56] have used patterning of polydimethylsiloxane (PDMS) to create ferroelectret structures for harvesting. Moulds were formed by 3D printing and used for moulding PDMS which were subsequently detached and plasma bonded to a dense PDMS layer to form the voids for corona poling. A further development of the process 
[57] used PVA to coat the inner voids to improve the degradation of polarisation over time.
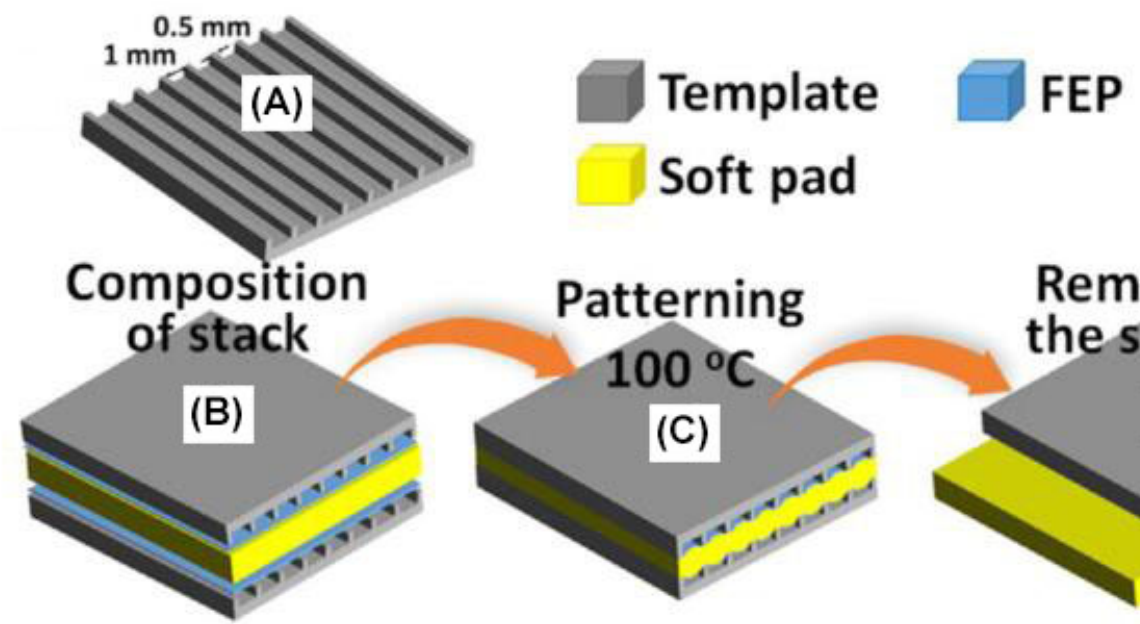

\section{SEM images}

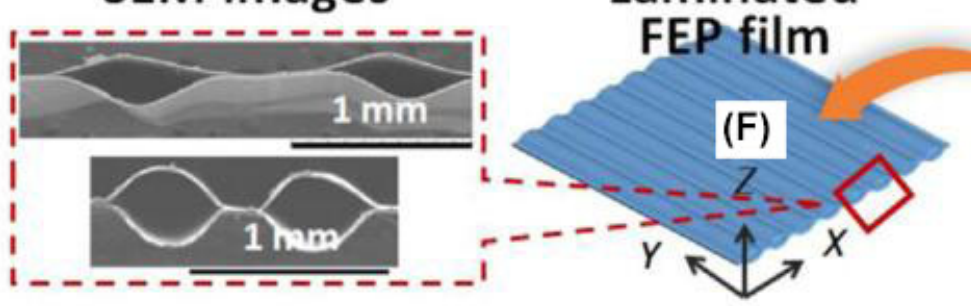

Removal of the soft pad

(D)

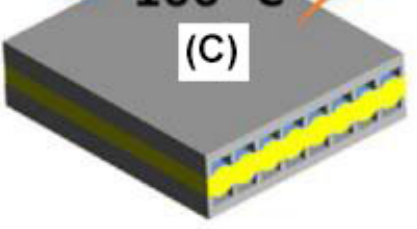

Laminated

(F)
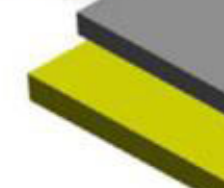

Fis

Fusion bonding $320^{\circ} \mathrm{C}$

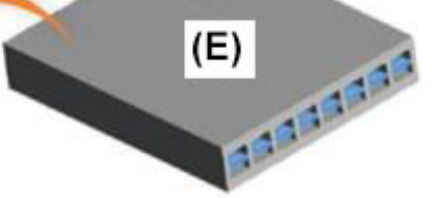

Fig. 6 Schematic of preparation of laminated FEP ferroelectret films. A is the template used in this procedure. $B$ is the composition of a stack used for the preparation. $C, D$, and $E$ are the steps of hot pressing at an elevated temperature of $100^{\circ} \mathrm{C}$, removal of the soft pad and fusion bonding above the melting point of FEP, respectively. $\mathrm{F}$ is a view of the completed FEP film. [4] Copyright 2018, Elsevier.

Patterning to form PDMS based ferroelectrets for energy harvesting has also been reported by Kachroudi et al. [58-60]. A low-cost method was developed to create a mould to form microcavities. A photomask was initially bonded to a photosensitive film (Riston MM540), see Fig. 7-1, and then inserted in a UV lithography system to cure, Fig. 7-2. The photosensitive film was then developed to form moulds with a height of $40 \mu \mathrm{m}$ and features with a diameter of $100 \mu \mathrm{m}$. A structured cellular PDMS layer was then formed by spin coating PDMS onto the mould, as in Fig. 7-4. After dicing to size (Fig.7-5.) bulk/dense PDMS was plasma bonded to the PDMS moulded section to create the cellular structure; Fig. 7-6. Clearly the use of three-dimensional printing or lithography provides significant scope to create uniform and highly designed pore geometries. 
(A) Mold preparation

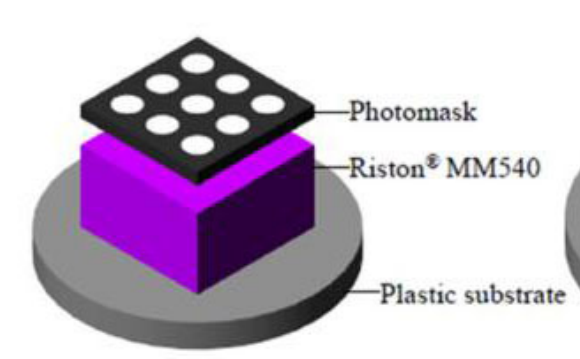

(D) Spin-coating of PDMS

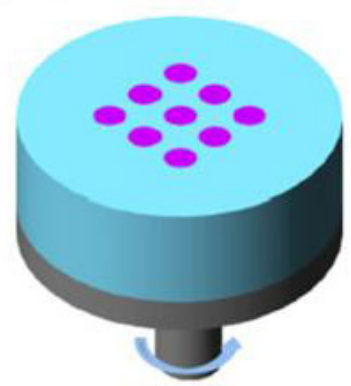

(B) UV-Lithography

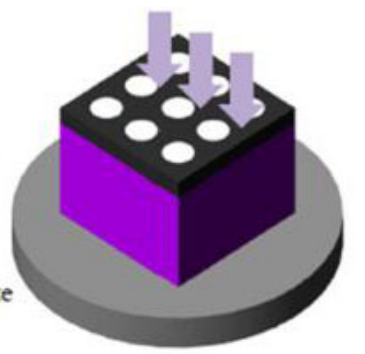

(E) Stripping and dicing

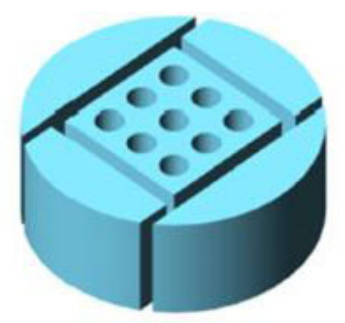

(C) Development

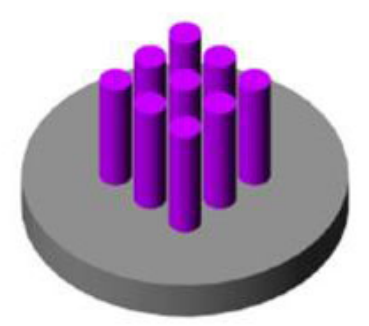

(F) Plasma bonding

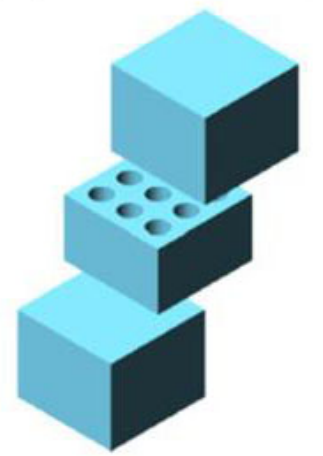

Fig. 7 Fabrication process flow of the micro-structured PDMS ferroelectret material. [60] (Copyright 2015 IOP Publishing)

\subsubsection{Self-poled systems}

One advantage of using PVDF and its related copolymers it that it can provide additional functions to a ferroelectret due to its inherent polar C-F bonds and the potential for spontaneous generation of electroactive $\beta$ - or $\psi$ - crystal phases. The foaming or incorporation of nanoparticles can further induce the formation and orientation of the polar crystal phases, and it has been reported that this can contribute to a 'self-poled effect', which eliminates the need for electrical poling or mechanical stretching processes. Mandal et al.[61] has developed metal particle filled porous polymer membranes for self-powered nanogenerators based on this mechanism; see Fig. 8A. 

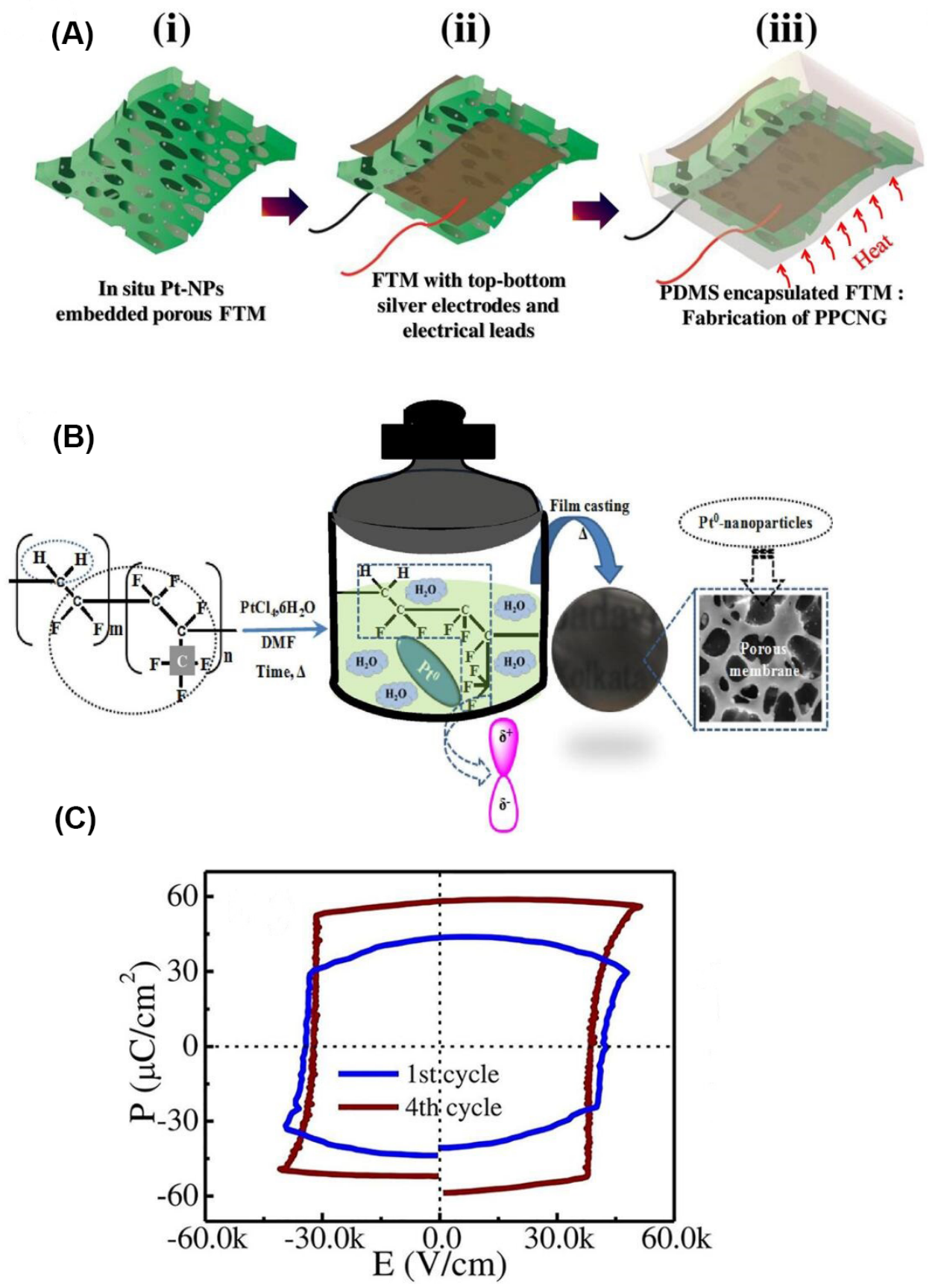

Fig. 8. (A) Fabrication of porous P(VDF-HFP) based composite membrane nanogenerators, where in (i) in situ Pt-NPs embedded porous membrane is shown, (ii) top and bottom silver electrode printing process and connection of two electrical leads, (iii) PDMS encapsulating process. (B) Schematic of the mechanism of pore formation, and a SEM image of the morphology of the membrane. (C) Polarisation-Electric field loop of porous P(VDF-HFP) based composite. Reprinted from [61] , with the permission of AIP Publishing.

Self-polarised porous poly(vinylidenefluoride-co-hexafluoropropylene) P(VDF-HFP) based composite membranes were prepared using the three steps shown in Fig. 8A 
$[61,62]$. Composites of P(VDF-HFP) containing different amounts of platinum salt $\left(\mathrm{H}_{2} \mathrm{PtCl}_{6} \cdot 6 \mathrm{H}_{2} \mathrm{O}\right)$ were prepared by solution casting to form films with a thickness of $\sim 150 \mu \mathrm{m}$ (Fig. 8(a-i)). Silver electrodes were then painted on the top and bottom side of the film and then conducting wires were attached to both electrodes using silver paste and dried at $60{ }^{\circ} \mathrm{C}$ (Fig. 8 (a-ii)). The films were then encapsulated with poly (dimethylsiloxane) (PDMS) layers and cured to form flexible nanogenerators (Fig. 8 (aiii)). As shown in Fig. 8b, the coordination of the water molecules of $\mathrm{H}_{2} \mathrm{PtCl}_{6} \bullet 6 \mathrm{H}_{2} \mathrm{O}$ with the solvent dimethylformamide (DMF) and subsequent evaporation of water at high temperature are thought to be responsible for the formation of prolate spheroid shaped micro-pores. The use of 2 wt\% of platinum nanoparticles (Pt-NPs) leads to a material with $85 \%$ porosity, with a $1.3 \mu \mathrm{m}$ average diameter and an average height of $4.25 \mu \mathrm{m}$. In addition, the in situ formed Pt-nanoparticles induced the formation of an $85 \%$ fraction of electroactive $\beta$ - and $\gamma$ - phases. The ferroelectret nature of the polymer membranes are shown by the square shaped hysteresis loop in Fig. 8c, with piezoelectric coefficient $d_{33} \sim-836 \mathrm{pC} / \mathrm{N}$, and piezoelectric voltage coefficient $g_{33} \sim$ $0.035 \mathrm{Vm} / \mathrm{N}$. The as prepared nanogenerators exhibited an open-circuit voltage of $6 \mathrm{~V}$ and short-circuit current of $6.4 \mu \mathrm{A}$ under repeating finger pressing at a stress of 1.1 MPa [61], indicating the hybrid polymer nanocomposite film is suitable for flexible piezoelectric-based energy harvesters. The coupling of both the self-aligned ferroelectric $\beta$-phase and the trapped charges in the pores is thought to be responsible for the superior properties of the composites. Following the same mechanism, $\mathrm{ZnO}$ nanoparticle filled $\mathrm{P}(\mathrm{VDF}-\mathrm{HFP})$ porous membranes were prepared containing $88 \%$ ferroelectric phases, with a high longitudinal piezoelectric charge coefficient, $d_{33} \sim$ $15.2 \mathrm{pC} / \mathrm{N}$ and a high voltage coefficient $g_{33} \sim-0.076 \mathrm{Vm} / \mathrm{N}$. The flexible nanogenerator produced an open-circuit voltage of $9 \mathrm{~V}$ and short circuit current of 1.3 $\mathrm{mAcm}^{-2}$ under a repeated mechanical impact stress of $0.36 \mathrm{MPa}$ [63]. A method to create ferroelectret type architectures for energy harvesting without the need for a poling process was also described by Xu et al. [64]. In this case a cellular ferroelectric polymer (PVDF) had its voids filled with a solid polyelectrolyte (Nafion), which is formed by solution casing a PVDF-Nafion blend. The composite material is then subjected to high pressure crystallisation to form the ferroelectric $\beta$-phase of PVDF. The piezoelectric potential of the PVDF during the application of stress is 
thought to lead to the motion of ions in the Nafion filled pores to enhance electromechanical performance for harvesting. The reasons for the piezoelectric response of the unpoled PVDF is of interest to explore, for example the application of high stress during processing.

\section{Characterisation and properties of ferroelectrets for energy harvesting}

The nature of the ferroelectret material, the manufacturing methods (as outlined in section 2), together with the achieved pore characteristics, piezoelectric coefficients, dielectric properties and the energy generated from ferroelectret based energy harvesters are summarised in this section and are shown in Table 1.

\subsection{Typical microstructure of ferroelectrets used for energy harvesting}

Mellinger et al. [65] indicated that the piezoelectric properties strongly depend on the pore morphology of the ferroelectret material in terms of pore height, pore width and pore shape, in particular along the poling direction; this is also shown in Fig. 3A. Scanning electron microscopy (SEM) and field-emission scanning electron microscopy (FE-SEM) have been employed as methods to examine the pore morphology, including for energy harvesting applications, revealing a range of architectures and pore sizes; see Table 1.

Wang et al. [55] employed commercially fibrous PTFE film with internal air bubbles with a pore height and pore width of 1-5 $\mu \mathrm{m}$ for charge storage after corona poling; the structure is shown in Fig. 9 (A). Such a pore size is almost at the minimum breakdown voltage, see Fig. 3B. Moreover, spherical pores were obtained in spongelike $\mathrm{P}(\mathrm{VDF}-\mathrm{HFP})$ film with the maximum pore size of $\sim 5 \mu \mathrm{m}$ [66]. A similar sponge-like structure in P(VDF-HFP) film was found by employing ZnO nanoparticles as the pore former, where the average pore diameter was $0.9 \mu \mathrm{m}$, as seen in the field emission SEM (FE-SEM) image of Fig. 9 (B) [63]. Moreover, Sujoy et al. [61, 62] fabricated a porous $\mathrm{P}(\mathrm{VDF}-\mathrm{HFP})$ film with prolate spheroid-shaped microscale voids whose average pore diameter and pore height were 1.55 and $4.5 \mu \mathrm{m}$, respectively, as observed in FESEM image of Fig. 9 (C). 
In addition to conventional pore shapes described above, another microstructure that exhibited a flower-like morphology was fabricated in an Yb-incorporated PVDF film, and the pore size was less than $1 \mu \mathrm{m}$, as shown in the FE-SEM image of Fig. 9(D) [67]. Ma et al. [68] employed a commercial ferroelectret polypropylene (PP) film with a thickness of $38 \mu \mathrm{m}$ that exhibited a cellular microstructure with a smaller pore height of $<1 \mu \mathrm{m}$ and pore width of $\sim 2 \mu \mathrm{m}$. Unlike the above mentioned polymer-air ferroelectret composites, Nafion was used to fill the holes in PVDF with different ratios between PVDF and Nafion whose morphologies are in the SEM image of Fig. 9(E) [64], where the Nafion was subsequently removed by etching. No voids were obtained for pure PVDF and low concentrations of Nafion (Fig. 9(E)a and b), while the crystalline PVDF cells became larger (several micrometers) and more open and interconnected with greater Nafion concentrations (Fig. 9(E) c to f). 

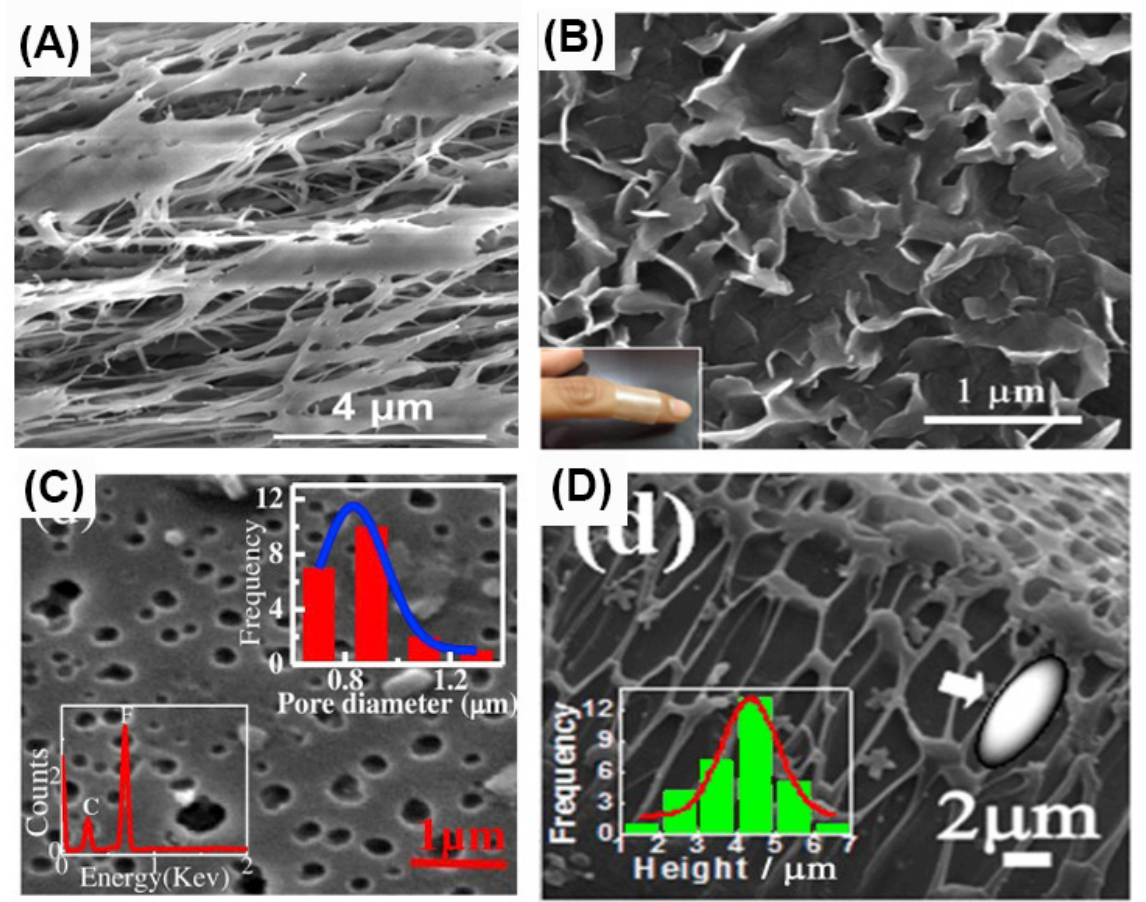

(E)

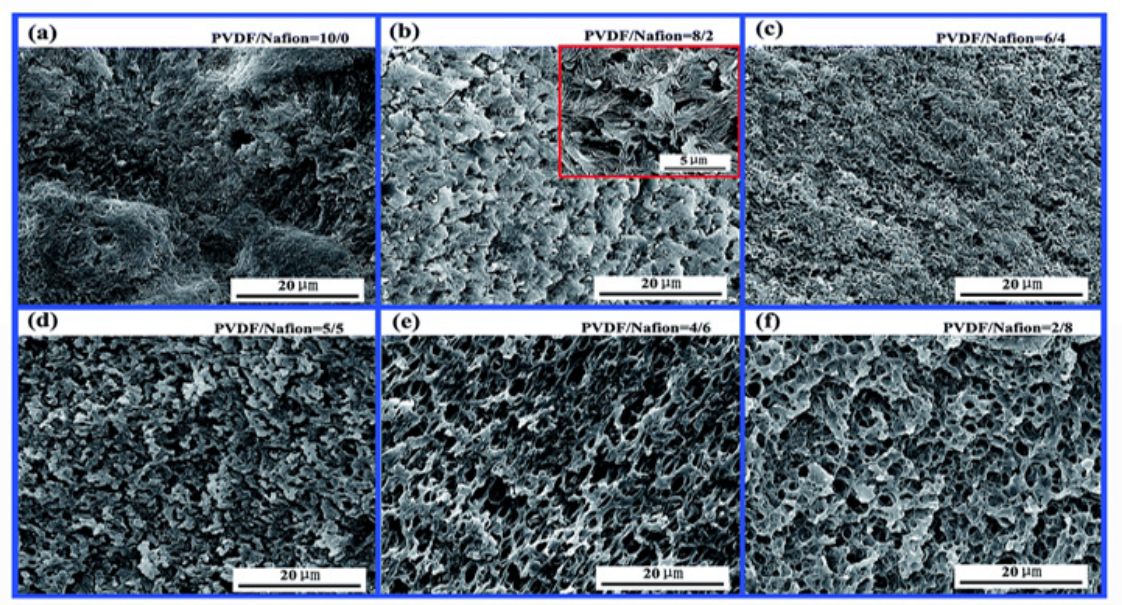

Fig. 9 SEM/FESEM images of the crossed section of the ferroelectret films with pore dimension a few microns. (A)f-PTFE layer [55], Copyright 2016 John Wiley and Sons, (B) PE film [63], Copyright 2017

Royal Society of Chemistry, (C) P(VDF-HFP) [61, 62], Copyright 2017 Elsevier, (D) Yb-PVDF [67],

Copyright 2015 John Wiley and Sons, (E) PVDF-Nafion composite samples with different compounding ratios [64], Copyright 2016 Elsevier.

In addition to the small-scale pores outlined above, pore lengths larger than tens of microns or even few hundreds of microns have been investigated for energy harvesting applications. Fig. $10(\mathrm{~A})[69,70]$ shows an SEM image of a ferroelectret foam manufactured from a polypropylene (PP). The thickness of the film is $\sim 70 \mu \mathrm{m}$ and it can also be seen that the lens-like pores are distributed homogeneously with a 
pore height of $\sim 10 \mu \mathrm{m}$, and pore width of $\sim 50 \mu \mathrm{m}$. Fig. 10(B) shows the microstructure of an as-received irradiation-crosslinked polypropylene (IXPP) foam with a thickness of $1.5 \mathrm{~mm}$. Round or hexahedron voids with a diameter of up to $300 \mu \mathrm{m}$ can be clearly observed in the SEM image $[11,71]$. After modification by a hot-pressing process for 20 min at $100{ }^{\circ} \mathrm{C}$ at a pressure of $15 \mathrm{MPa}$, the thickness of the IXPP reduced to 180 $\mu \mathrm{m}$ and disk-like pores can be observed via SEM, as shown in Fig. 10(C). Zhang et al. $[3,6,72,73]$ used a patterning and fusion bonding processes to stack two commercial fluoroethylenepropylene (FEP) films, of $12.5 \mu \mathrm{m}$ thickness, together to form a crosstunnel structure, as shown in Fig. 10(D). Other commercial films in terms of polyethylene terephthalate (PET), biaxially oriented polypropylene (BOPP) and ethylene vinyl acetate (EVA) have been used for further processing in Zhong et al.'s work $[33,42]$. With the aid of a stainless-steel mask with uniform convex and concave pattern, a laminated cellular bubble-like structure in the PET/BOPP film was formed. The regular and uniform air bubbles had a height of $\sim 300 \mu \mathrm{m}$ with a maximal width of $\sim 1.3 \mathrm{~mm}$, as shown in Fig. 10(E) [42]. A PET/EVA film with an arch-shaped air bubble with a height of 0.5-1 mm was achieved after hot pressing [33]. In addition, Kachroudi et al. [58] fabricated a microstructured layer of $40 \mu \mathrm{m}$ thickness, containing cylindrical micro-cavities of $100 \mu \mathrm{m}$ diameter and a pitch of $150 \mu \mathrm{m}$, shown in Fig. 10(F). By activating the chemical blowing agent, pore sizes ranging from 90 to $340 \mu \mathrm{m}$ were created in the polyethylene (PE) foam [74].

From the above discussion, and Table 1, it can be seen that to facilitate Townsend breakdown to create polarised pores for a ferroelectret energy harvester, pore heights of the ferroelectret polymers are of the order of microns to achieve low breakdown voltages, as shown in Fig. 3B where at atmospheric pressure ( $1 \mathrm{~atm})$ the $y$-axis corresponds to separation distance. The pore length varies considerably, ranging from few microns to hundreds microns, and there is a preference for elongated pores normal to the polarisation direction to reduce material stiffness and maximise area for charging. In real materials there is likely to be a range of pore heights, and therefore dielectric barrier discharge will occur at a range of voltages, leading to a tilting of the polarisation-electric field loop in Fig. 1B; this has similarities 
to tilting of the polarisation-field loop in ferroelectric ceramics since different domain types have different coercive fields.
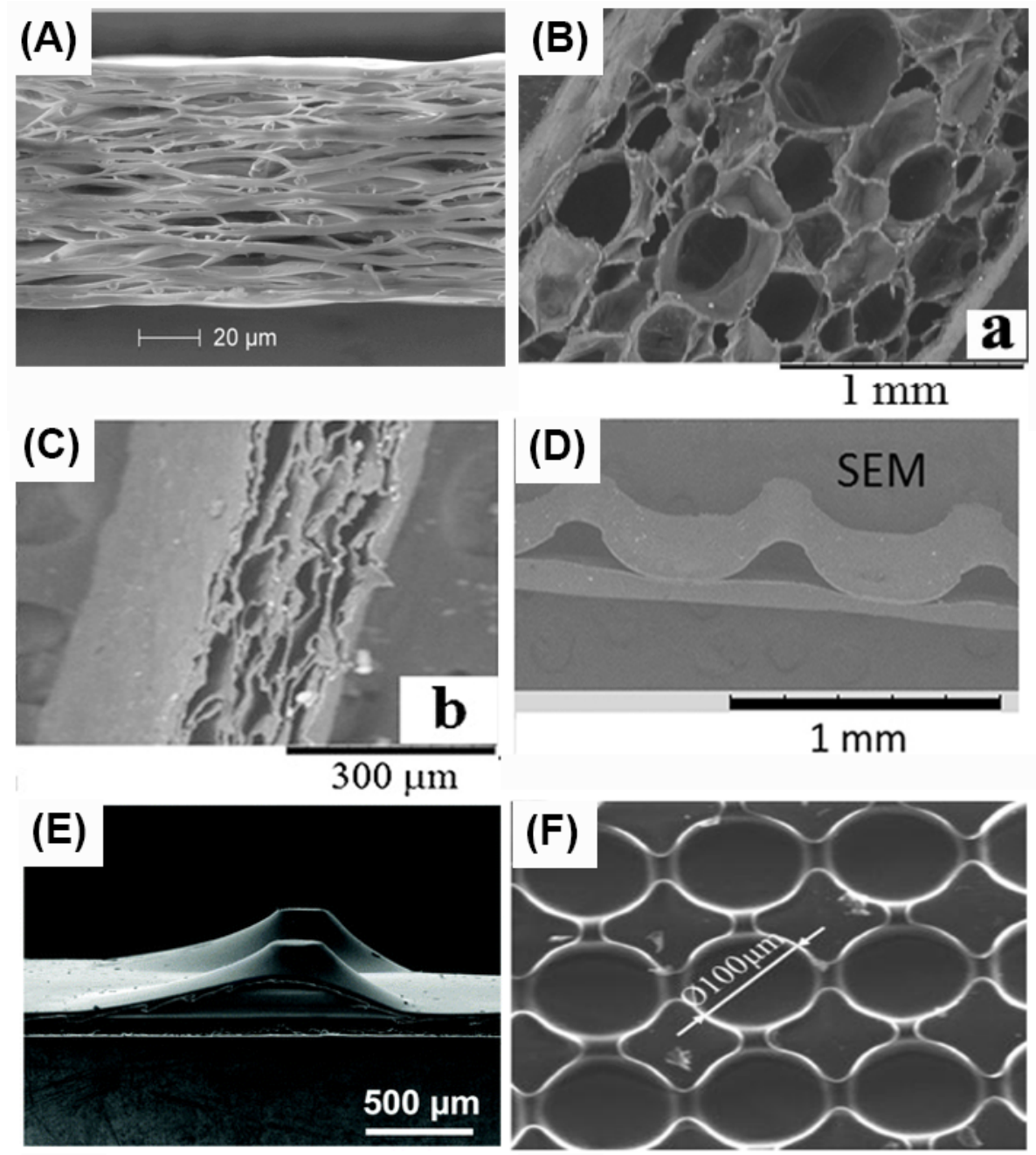

Fig. $10 \mathrm{SEM}$ /digital images of the cross section of the ferroelectret films with pore dimension larger than tens of microns. (A) cellular polypropylene film [69, 70],Copyright 1999 AIP Publishing, cross-linked polypropylene [11], (B) before and (C) after modification, (Creative Commons Attribution (CC BY) license) (D) fluoroethylenepropylene [3], Copyright 2014 AIP Publishing, (E) polyethylene terephthalate/polypropylene [42], Copyright 2016 RSC Publishing, (F) polyethylene terephthalate/ethylene vinyl acetate [58] Creative Commons Attribution 3.0 licence.

3.2 Piezoelectric coefficients and dielectric properties of ferroelectret energy harvesters

It is important to assess the electromechanical performance of the ferroelectret materials, this includes (i) the piezoelectric coefficient $\left(d_{33}\right)$ which is a measure of the 
charge generated per unit force in the polarisation direction, (ii) the relative permittivity $\left(\varepsilon_{r}\right)$, also termed the dielectric constant, which is used for evaluating the ability of a dielectric material to store energy in an electric field, (iii) the dielectric loss, which is an indicator of the dielectric material's energy dissipation ( $\tan \delta$ ) and (iv) electromechanical coupling factor $\left(k_{33}\right)$ which is used for calculating the conversion efficiency between electrical energy and mechanical energy in the active materials. Such properties have been commonly explored for energy harvesting systems, and can be measured via a quasi-static $d_{33}$ meter and an impedance analyser. The dynamic measurement of $d_{33}$ has also been conducted by sinusoidally accelerating the ferroelectret polymer with a mass on it by a shaker [75]. Figures of merit are also calculated from these material properties, such as Equation 1 for harvesting in $d_{33}$ mode and a figure of merit for sensing, which will be discussed later in the review.

Anton et al. [76-78] excited a ferroelectret PP foam by an electromagnetic shaker to test the dynamic piezoelectric coefficient $\left(d_{33}\right)$. A range of frequencies from $5 \mathrm{~Hz}$ to $1 \mathrm{kHz}$, using different masses $(100 \mathrm{~g}, 500 \mathrm{~g}$ and $1 \mathrm{~kg})$ at three acceleration levels $(0.1 \mathrm{~g}$, $0.3 \mathrm{~g}$ and $0.5 \mathrm{~g}$ ) were used during the test; such a test is of interest as it is similar to the conditions at which the material would be used as a vibrational energy harvester. The $d_{33}$ value remained relatively constant at $\sim 35 \mathrm{pC} / \mathrm{N}$ irrespective of the frequency and the mass applied, as shown in Fig. 11(A). However, different loading masses varied from $13 \mathrm{~g}$ to $23 \mathrm{~g}$ resulted in a reduced $d_{33}$ value, as shown in Fig. $11(\mathrm{~B})$, due to the reduced distance between the opposite implanted charges in the pore surfaces. A dynamic piezoelectric coefficient $\left(d_{33}\right)$ of IXPP ferroelectret film up to $650 \mathrm{pC} / \mathrm{N}$ at a frequency of $200 \mathrm{~Hz}$ was found via this approach [11]. 
(A)

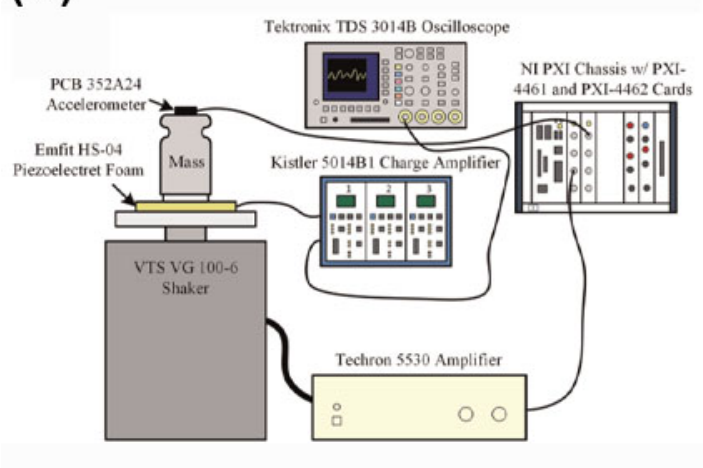

(B)

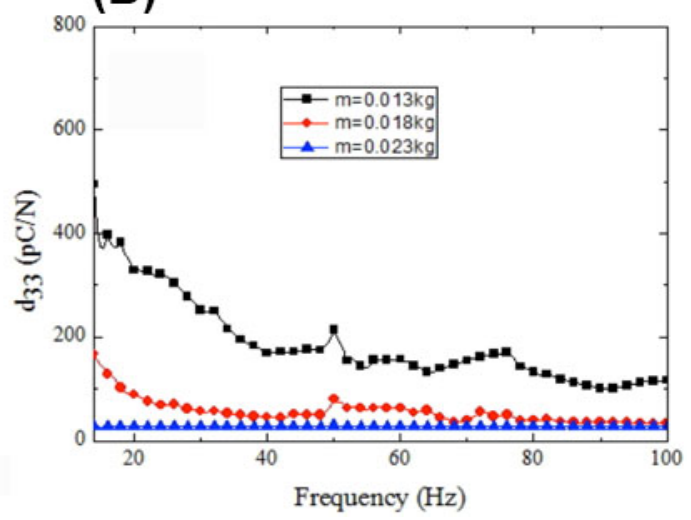

Fig. 11 (A) Experimental test setup for dynamic $d_{33}$ measurements [76, 77], Copyright 2014 SAGE, (B) dynamic piezoelectric response of PDMS piezo-electret for various loaded masses.

[79] Creative Commons Attribution - NonCommercial 4.0 International License.

In addition to the above measured dynamic piezoelectric coefficients, quasi-static characterisation of materials for ferroelectric energy harvesters have also been explored. Porous P(VDF-HFP) films with different additions of the hydrated magnesium salt exhibited a relative permittivty that decreased gradually as the frequency was increased from $1 \mathrm{kHz}$ to $1 \mathrm{MHz}$, but no $d_{33}$ was reported [66]. The magnesium-salt filler with $0.5 \mathrm{wt} . \%(\mathrm{Mg} 0.5)$ achieved a maximum value at a constant frequency, as seen in Fig. 12(A). Fig. 12(B) shows that the dielectric loss increased with an increasing amount of magnesium-salt filler. Comparable observations in Fig. 12(C) [63] were found in $\mathrm{ZnO}$ nanoparticle etched porous sponge-like P(VDF-HFP) film with a relative permittivity, $\varepsilon_{r} \sim 22.5$ and $\tan \delta \sim 0.07$ at $1 \mathrm{kHz}$, respectively. A multilayer ferroelectret with nine layers folded from a commercial PP film exhibited a $d_{33} \sim 290$ $\mathrm{pC} / \mathrm{N}$, compared to a $d_{33} \sim 220 \mathrm{pC} / \mathrm{N}$ for a single layer film. Meanwhile, a 10-layer ferroelectret film with $d_{33} \sim 300 \mathrm{pC} / \mathrm{N}$ was used to predict the power generated by vibration energy harvesting by simulation with different seismic masses and a number of film layers [80]; modelling strategies are described in more detail in Section 4. A relative permittivity of $\varepsilon_{r} \sim 1.2$ was reported in a stacked PP film for vibration-based energy harvesting by the same group [81]. 

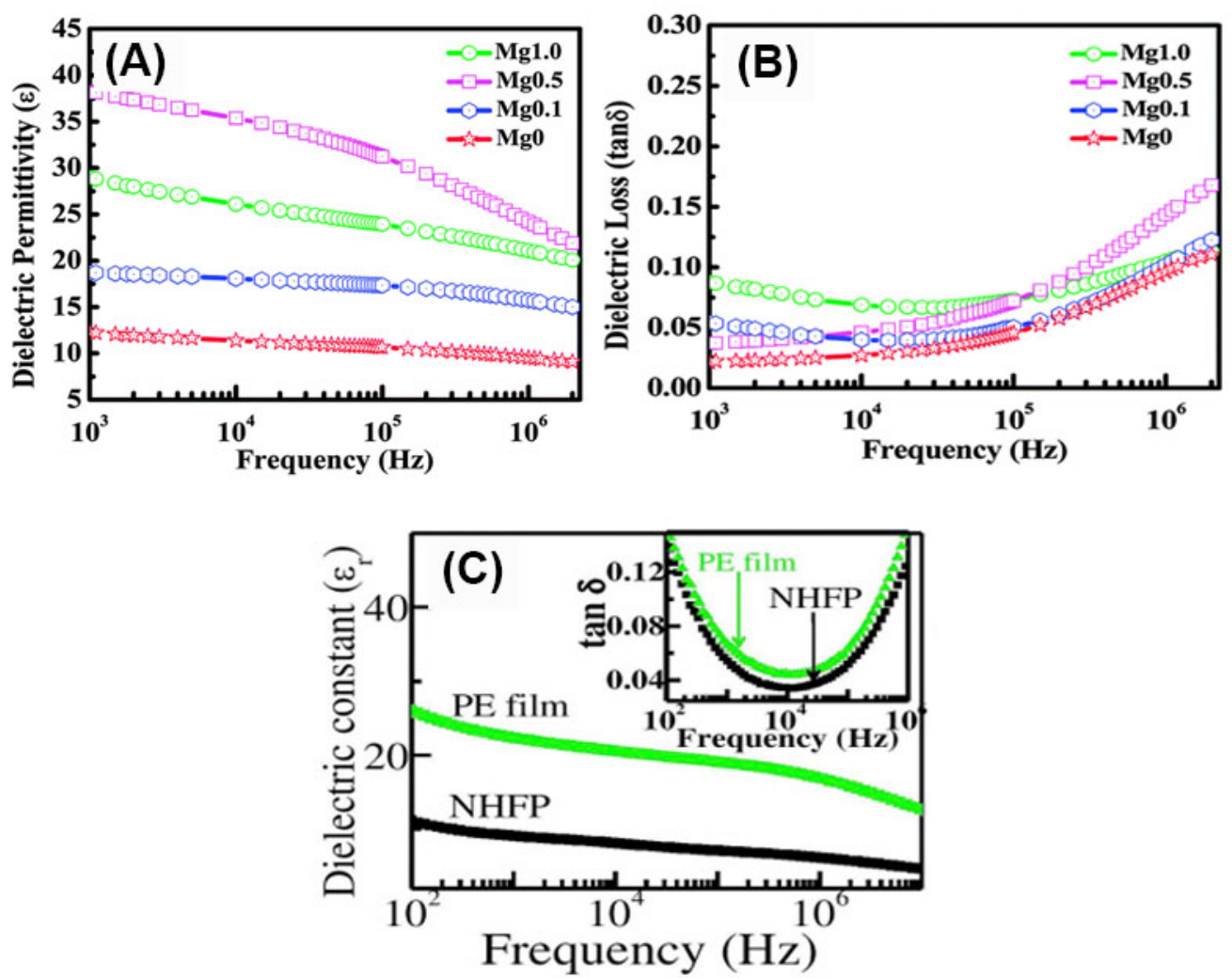

Fig. 12 Permittivity and dielectric loss of ferroelectret energy harvester. (A) Variation of dielectric constant and (B) loss as a function of applied frequency in P(VDF-HFP) films with Mg-salt addition \# from 0.1 to $1.0 \mathrm{wt}$ \% (Mg\#). [66] Reproduced with permission from The Royal Society of Chemistry. (C) frequency-dependent dielectric constant $\left(\varepsilon_{\mathrm{r}}\right)$ with loss tangent (tan $\delta$ ) (inset) of porous P(VDF-HFP) films [63]. Reproduced with permission of Elsevier.

Table 1 lists the electrical performance of some ferroelectret polymers under different temperatures and duration time. A comparable value of $d_{33} \sim 350 \mathrm{pC} / \mathrm{N}$ at $25^{\circ} \mathrm{C}$ was reported in a micro-structured PDMS by Kachroudi et al., with the temperature dependence of $d_{33}$, and the effects of the temperature on the dielectric loss [58]. A life-time and aging study on this micro-structured PDMS at room temperature was subsequently performed [59], and demonstrated a high stability with $90 \%$ of the piezoelectricity being maintained after 50 days. A piezoelectric coefficient $d_{33}$ of 200 $\mathrm{pC} / \mathrm{N}$ was reported in the porous polyethylene (PE) thick-foam designed and engineered for energy harvesting applications [74]. Shi et al. found that the $d_{33}$ of the ferroelectret polymer increased 1.5 times as compared to pure polydimethylsiloxane (PMDS) after the addition of polyvinyl alcohol (PVA) on the surface of PDMS. The PDMS/PVA ferroelectret also retained $\sim 80 \%$ of its initial $d_{33}$ value, compared with 
$\sim 40 \%$ for pure PDMS over $72 \mathrm{~h}$ [57]. Compared to the original commercially and biaxially oriented PP film with $d_{33}$ of $19 \mathrm{pC} / \mathrm{N}$, an improved $d_{33} \sim 205 \mathrm{pC} / \mathrm{N}$ was achieved after a rapid deflation process, which was maintained at a $d_{33} \sim 205 \mathrm{pC} / \mathrm{N}$ for weeks and was stable and repeatable below $60{ }^{\circ} \mathrm{C}$ [51]. This demonstrates that by appropriate materials selection and design there is potential to achieve long-term stability and low aging rates for energy harvesting.

Table 2 Electromechanical properties of piezoelectric coefficient and permittivity of ferroelectrets used for harvesting; with different temperatures and durations.

\begin{tabular}{|l|l|l|l|l|}
\hline Material & Temperature & Duration & $\begin{array}{l}\text { Electromechanical } \\
\text { coupling factor, } k_{33}\end{array}$ & $\begin{array}{l}\text { Piezoelectric } \\
\text { coefficient, } d_{33}, \mathrm{pC} / \mathrm{N}\end{array}$ \\
\hline PDMS [58] & $-20-85^{\circ} \mathrm{C}$ & - & $0.03-0.05$ & - \\
\hline PDMS [59] & Room temperature & $0-50$ days & - & $340-350$ \\
\hline $\begin{array}{l}\text { PDMS/PVA, } \\
{[57]}\end{array}$ & Room temperature & $0-72 \mathrm{~h}$ & - & $90-110$ \\
\hline $\begin{array}{l}\text { Polypropylene } \\
{[51]}\end{array}$ & $30-60^{\circ} \mathrm{C}$ & $0-6$ weeks & - & $\sim 200$ \\
\hline
\end{tabular}

In addition to the above mentioned ferroelectrets with moderate piezoelectric coefficient of several hundreds $\mathrm{pC} / \mathrm{N}$ for energy harvesting, it is of interest to note a polyethylene terephthalate-ethylene vinyl acetate (PET/EVA)-based ferroelectret film that reached a very high $d_{33} \sim 6300 \mathrm{pC} / \mathrm{N}$, which was maintained in a narrow range around the original value for six weeks in indoor atmosphere [33]. A large $d_{33} \sim$-686 $\mathrm{pC} / \mathrm{N}\left(-836 \mathrm{pC} / \mathrm{N},([61])\right.$, and an ultrahigh $\varepsilon_{r} \sim 2678$ and $\tan \delta \sim 0.79$ at $1 \mathrm{kHz}$ [62], were obtained in the platinum nanoparticles embedded porous P(VDF-HFP) film, owing to the charge trapping sites produced from self-polarized $\beta$-phase and the micropores in the film, which resulted in a $d_{33} \sim-362.9 \mathrm{pC} / \mathrm{N}, \varepsilon_{r}>50000$ and $\tan \delta \sim 7.8$ at $1 \mathrm{~Hz}$ in the $\mathrm{Yb}^{3+}$ assisted PVDF film [67]; it is of interest to note that high losses and high permittivity values can often be associated with the presence of electrical conductivity [82] and is worth examining in more detail. Fig. 13(A) shows a $d_{33}$ from few hundreds up to $3700 \mathrm{pC} / \mathrm{N}$ under the peak voltage applied to the FEP film, and the antiresonance frequency of $86 \mathrm{kHz}$ was found in Fig. 13(B) [3]. Comparable values of $d_{33} \sim$ 
$1000 \mathrm{pC} / \mathrm{N}$ were found in their research focusing on using different types of templates for pore production $[6,72]$.

(A)

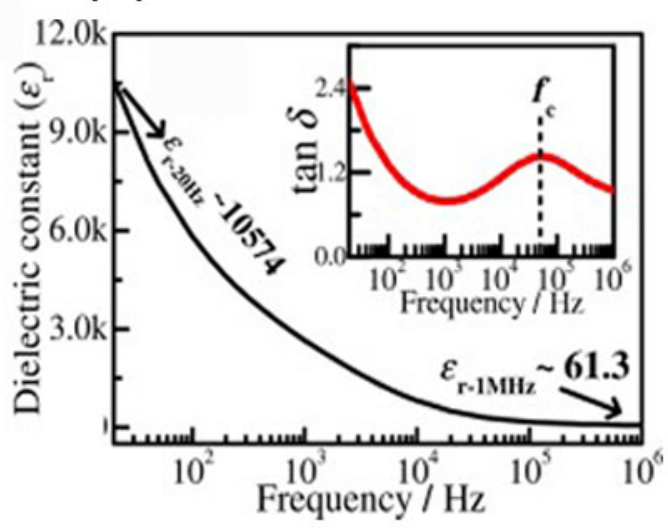

(B)

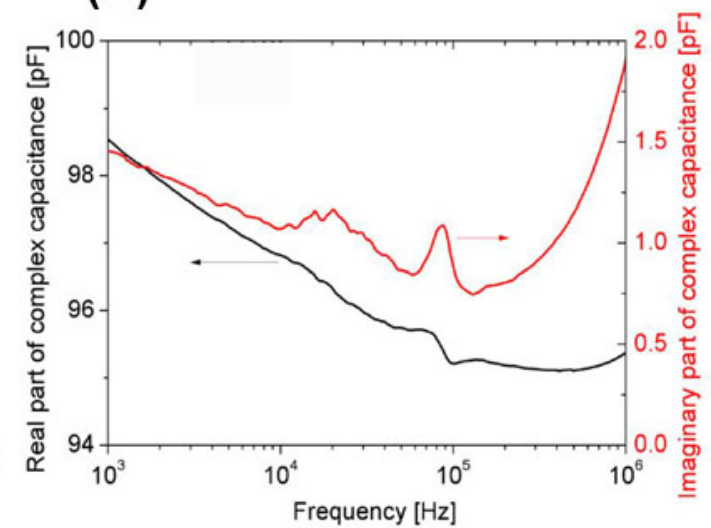

Fig. 13 Electromechanical properties in terms of piezoelectric coefficient and dielectric response. (A) frequency-dependent dielectric constant $\left(\varepsilon_{\mathrm{r}}\right)$ with loss tangent $(\tan \delta)$ (inset) [62], Copyright 2015 John Wiley and Sons. (B) dielectric resonance spectrum of cross-tunnel FEP films [3], Copyright 2014 AIP Publishing.

\subsection{Phase content}

Due to the semi-crystalline nature of the PVDF, four main crystalline phases $(\alpha, \beta, \gamma$ and $\delta$ ) with different electromechanical performances are present depending on the crystallization conditions, the size of the crystalline regions, and the degree of crystallization. As stated, the piezoelectric properties of a ferroelectret originate from the charged pores; however if combinations of ferroelectric and ferroelectret responses are potentially present, the nature of the polymeric phases is of interest. Phase identification of PVDF, its copolymer and PVDF-based composites can be normally confirmed and distinguished by X-ray Diffraction Analysis (XRD) and Fourier Transform Infrared (FTIR) spectroscopy.

Ghosh et al. proved the presence of the uniaxially oriented polar $\beta$-phase in Yb-PVDF composite film with the evidence of the vibration band at $1277 \mathrm{~cm}^{-1}$ in the FT-IR spectra, compared with the pure PVDF composed of the non-polar $\alpha$-phase under the same condition, as shown in Fig. 14(A) and (B) [67]. An in-situ synthesised platinum (Pt) nanoparticle doped porous $\mathrm{P}(\mathrm{VDF}-\mathrm{HFP})$ membrane was prepared, where the $\mathrm{Pt}$ phase was confirmed by diffraction peaks in XRD, along with the size of $40 \mathrm{~nm}$ estimated from Debye-Scherrer equation based on the data from XRD, as shown in 
Fig. 14(C). Correspondingly, it can be seen from Fig. 14(D) that the presence of the electroactive and polar $\gamma$ - and $\beta$-phases were identified from the FT-IR spectra, while the non-polar $\alpha$-phase was detected in the pure P(VDF-HFP) sample [67]. In a PVDFNafion composite with different ratios between PVDF and Nafion, a continuous increase in the content of the ferroelectric $\beta$-phase was evident in the wide-angle $X$ ray diffraction (Fig. 14(E)) and FT-IR (Fig. 14(F)) spectra when Nafion concentration decreased, as long as a relatively low contents of $\alpha$ - and $\psi$-phases were identified. Adhikary et al. fabricated a sponge-like P(VDF-HFP) film assisted by different additions of the hydrated magnesium salt. Almost all of the nonpolar $\alpha$-phase characteristic peaks were crystallographically transformed to ferroelectric polar $\beta$ - and $\psi$-phases, as observed from the FT-IR spectra, compared with the predominant $\alpha$ phase in the pure P(VDF-HFP). This is shown in Fig. 14(G). XRD results in Fig. 14(H) indicated that electroactive $\beta$ and $\gamma$ phases were formed in a Mg-salt utilized film while the $\alpha$-phase is diminished. The Mg-salt was regarded as a nucleation site to enhance the polarphase transformation in P(VDF-HFP) [66]. 

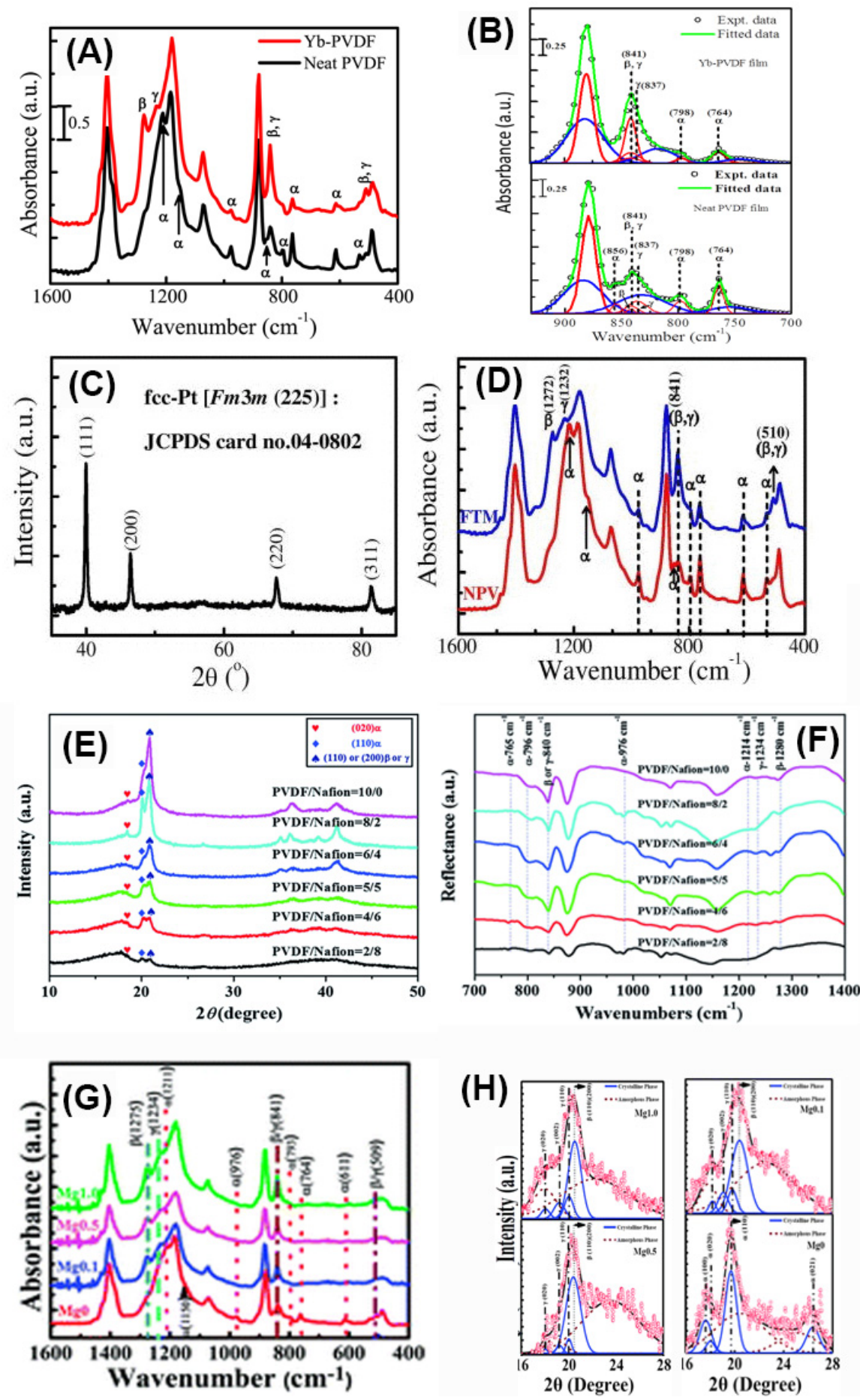

Fig. 14 (A) FT-IR spectra of pure PVDF and Yb-PVDF composite films in the wavenumber range of $1600-400 \mathrm{~cm}^{-1}$ to identify the crystalline polymorph [67], (B) Curve deconvolutions of XRD pattern in $2 \theta \sim 15-30^{\circ}$ range have been performed to represent the crystal reflections from the pure PVDF (lower panel) and Yb-PVDF (upper panel) films. [67] Copyright 2016, Elsevier, (C) XRD pattern of FTM ensures the diffraction peaks from Pt-NPs. [61], (D) FT-IR spectra of FTM and NPV from 1600 to $400 \mathrm{~cm}^{-1}$ frequency region [61], Copyright 2016, AIP Publishing, (E)

WAXD and (F) ATR-FTIR results of the PVDF-Nafion composite samples with different compounding ratios [64], Copyright 2017 Royal Society of Chemistry, (G) FT-IR spectra of Mg\# (where, \#: 0, 0.1, 0.5, 1.0) films from 1600 to $400 \mathrm{~cm}^{-1}(\mathrm{H})$ The XRD pattern of Mg\# (where, \#: $0,0.1,0.5,1.0$ wt.\%) films and its curve deconvolution [66]. Copyright 2015 Royal Society of Chemistry. 
Although it is not necessary for ferroelectret materials to be inherently piezoelectric or ferroelectric, ferroelectric polymers such as PVDF, its copolymers (P(VDF-HFP)) and PVDF-based composite have been fabricated and optimised as ferroelectret materials $[61,64,66,67]$, whose ferroelectric responses can potentially contribute to an additional effect on designing mechanically vibration-based energy harvester via piezo-, pyro-, and ferro-electric effects. Combining ferroelectric and ferroelectret effects is an interesting route to overcome the poor pyroelectric properties of ferroelectrets; for example if a combination of both piezoelectric and pyroelectric properties is desirable.

\section{Modelling of ferroelectrets}

This section describes approaches to model ferroelectret materials to understand the impact of microstructure and vibration conditions during energy harvesting on key properties such as permittivity, piezoelectric coefficients and frequency response.

\subsection{Relative permittivity (dielectric constant)}

In terms of the dielectric properties, a Lichtenecker mixing rule has been employed for porous materials [83], see Equation 2;

$k_{t}^{\alpha}=k_{s}^{\alpha}\left(1-V_{g}\right)+k_{g}^{\alpha} V_{g}$

where $k_{s}$ and $k_{g}$ are the dielectric constants of the solid and gaseous phases, respectively, $V_{g}$ is the volume fraction of voids (i.e., the porosity), and $\alpha$ is a parameter that determines the type of rule of mixtures. If $\alpha=-1$, then the series mixing rule can be used which represents a lower limit of the dielectric constant as shown by the dotted line in Fig. 15: $\frac{1}{k_{t}}=\frac{V_{g}}{k_{g}}+\frac{V_{s}}{k_{s}}$. If $\alpha=1$, then the parallel mixing rule is used which represents the upper limit of the dielectric constant as shown by the solid line in Fig. 13: $k_{t}=k_{s}\left(1-V_{g}\right)+k_{g} V_{g}[83]$. 


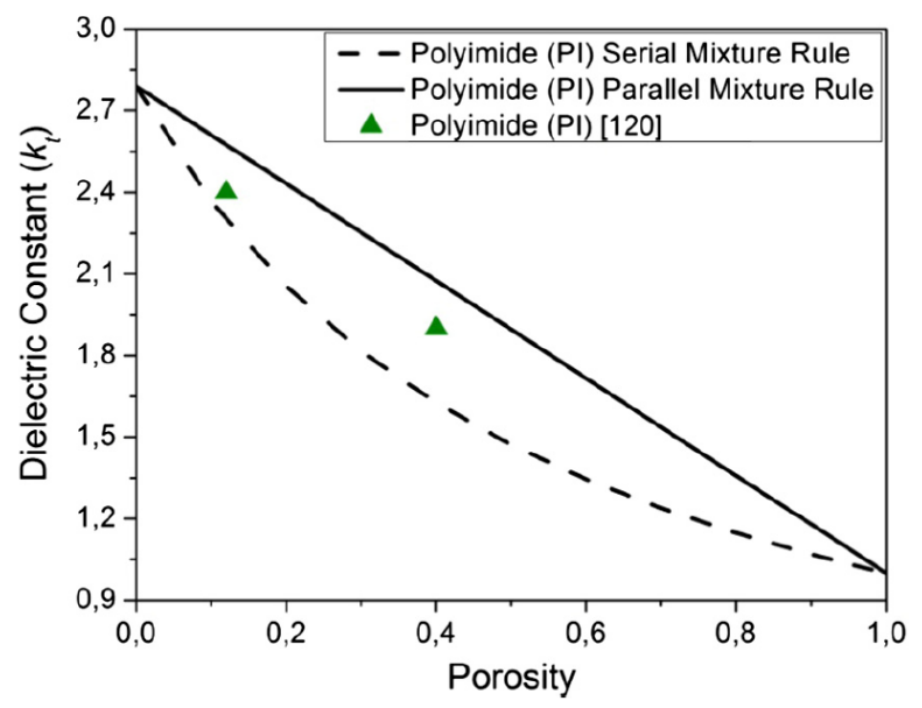

Fig. 15 Variation of dielectric constant with porosity and series and parallel mixing rules. [84] Reprinted with permission from Elsevier.

\subsection{Piezoelectric properties of ferroelectrets}

As stated, ferroelectrics exhibit a spontaneous polarization that can be reversed by an applied electric field. However, there are clear differences in origin of the piezo- and pyro-electric effects between ferroelectret cellular polymers and ferroelectric polymers, as shown in Fig. 3. Due to the anisotropy of the lens shaped voids and the low symmetry of the charge distribution, high piezoelectric sensitivity is primarily in the thickness direction (determined by $d_{33}$ coefficient) and is typically two orders of magnitude larger than the in-plane piezoelectric sensitivity (determined by $d_{31}$ and $d_{32}$ coefficients). This is in contrast to ferroelectric materials where $d_{33} \sim 2 d_{31}$. The electromechanical response of the cellular ferroelectrets has been described in detail by Sessler et al. and Paajanen et al. $[70,85]$ Since the lateral dimensions of the voids are much larger than their vertical dimensions, as in Fig.3, a simplified model has been developed to determine the piezoelectric properties of ferroelectrets (see schematic in Fig. 16). The model consists of alternating polymeric and air layers with a thicknesses $s_{1 i}$ and $s_{2 j}$, respectively, with $i=1,2, \cdots, n$ and $j=1,2, \cdots, n-1$, where $n$ is the total number of solid layers. The permanent charges on the two sides of each air gap that are formed as a result of Townsend discharge and are assumed to be equal in magnitude and are assumed to originate from dielectric barrier discharge in the air gap during the poling process. 


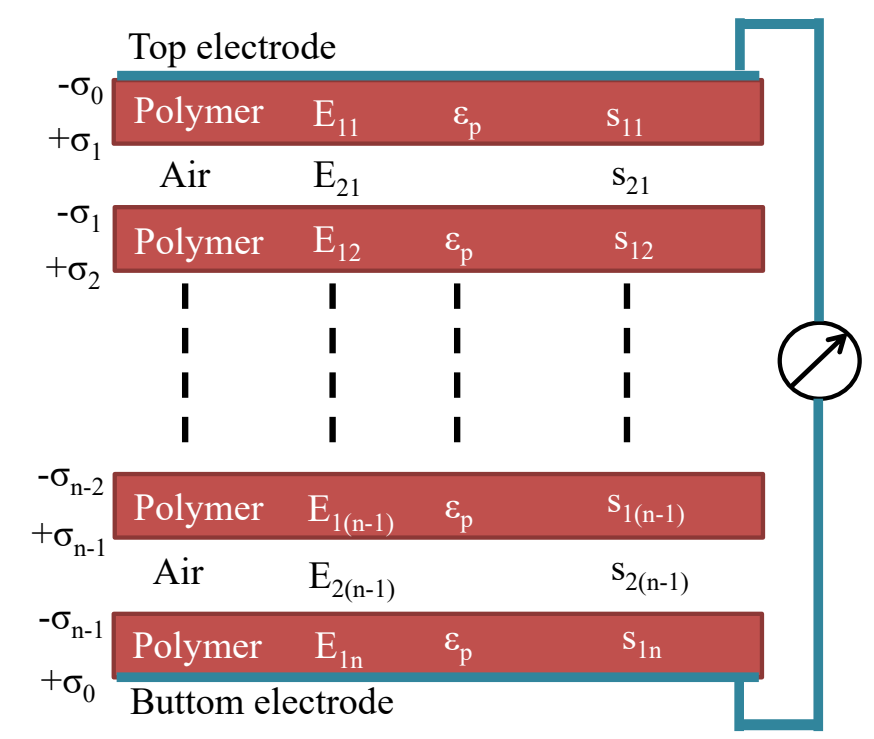

Fig. 16 Simplified layer model for ferroelectrets with alternating polymer and air layers (according to references. $[70,85])$. Charges of opposite polarity are generated during dielectric barrier discharge and deposited on the top and bottom air polymer interfaces, respectively. Reproduced with the permission from. [70, 85], Copyright 1999 AIP Publishing.

The electric field in the polymer and air are calculated using Gauss's and Kirchhoff's laws. For the upper and lower electrodes, Gauss' law is:

$$
\begin{gathered}
-\varepsilon_{0} \varepsilon_{p} E_{11}=-\sigma_{0} \\
\varepsilon_{0} \varepsilon_{p} E_{1 n}=\sigma_{0}
\end{gathered}
$$

and

For the upper and lower interfaces of the $j^{\text {th }}$ air gap,

$$
\begin{aligned}
& \text { and } \quad-\varepsilon_{0} E_{2 j}+\varepsilon_{0} \varepsilon_{p} E_{1 j}=-\sigma_{j} \\
& -\varepsilon_{0} \varepsilon_{p} E_{1(j+1)}+\varepsilon_{0} E_{2 j}=-\sigma_{j}
\end{aligned}
$$

Under short-circuit conditions, Kirchhoff's $2^{\text {nd }}$ law is:

$$
\sum_{i} s_{1 i} E_{1 i}+\sum_{j} s_{2 j} E_{2 j}=0
$$

Equations 5 and 6 lead to: 


$$
\begin{aligned}
& E_{11}=E_{12}=\ldots=E_{1} \\
& E_{2 j}=\varepsilon_{p} E_{1}-\frac{\sigma_{j}}{\varepsilon_{0}}
\end{aligned}
$$

Substituting equations (8) and (9) into equation (7):

$$
\begin{aligned}
& E_{1}=\frac{\sum_{j} s_{2 j} \sigma_{j}}{\varepsilon_{0} s_{1}+\varepsilon_{0} \varepsilon_{p} s_{2}} \\
& E_{2 i}=\frac{\varepsilon_{p} \sum_{j} s_{2 j} \sigma_{j}}{\varepsilon_{0} s_{1}+\varepsilon_{0} \varepsilon_{p} s_{2}}-\frac{\sigma_{i}}{\varepsilon_{0}}
\end{aligned}
$$

where, $s_{1}=\sum_{i} s_{1 i}$ and $s_{2}=\sum_{j} s_{2 j}$ are the combined total thickness of the polymer and air layers, respectively. When a pressure $p$ is applied to the ferroelectret, change in thickness are assumed to be a result of compression in layers of air. Therefore, the electrode charge varies according to $\frac{\partial \sigma_{0}}{\partial s_{2}}$, and if it is assumed that $\frac{\partial s_{2 j}}{\partial s_{2}}=\frac{s_{2 j}}{s_{2}}$, then:

$$
\frac{\partial \sigma_{0}}{\partial s_{2}}=\frac{\varepsilon_{p} s_{1}}{\left(s_{1}+\varepsilon_{p} s_{2}\right)^{2}} \sigma_{e f f}
$$

where, $\sigma_{e f f}=\frac{\sum_{j} s_{2 j} \sigma_{j}}{s_{2}}$ is the polarisation in the ferroelectret. From the stress-strain relationship $\frac{\Delta s_{2}}{s}=\frac{p}{Y}$, where, $s=s_{1}+s_{2}$ and $Y$ is the foam Young's modulus, the $\mathrm{d}_{33}$ piezoelectric coefficient is given by:

$$
d_{33}=\frac{\varepsilon_{p} s}{Y} \frac{s_{1} \sigma_{e f f}}{\left(s_{1}+\varepsilon_{p} s_{2}\right)^{2}}
$$

This provides the piezoelectric coefficient as a function of permittivity, Young's modulus, polarisation level and pore and air geometry, although more detailed models may be of interest to further optimise pore geometry and harvesting figures of merit, such as Equation 1.

\subsection{Electro-mechanical models}


Several electro-mechanical models to study the energy harvesting capability of ferroelectret materials have been reported, in particular the frequency dependence of the harvester response. These models are typically developed to predict the electrical output of a ferroelectric specimen when subjected to a mechanical excitation. An early model was developed by Lindner et al. [24] and consisted of negatively and positively charged particles connected by springs with different spring constants. More recently, Kärki and Lekkala [86] developed a lumped-parameter model for ferroelectret polymers based on an equivalent circuit to estimate the electro-mechanical coupling factor.

While quasi-static loading conditions have been studied [87]), dynamic modeling (vibratory excitation and dynamic response) are particularly appealing for vibration energy harvesting purposes. These models vary according to the design of the harvester being investigated, however a review of the pertinent literature reveals that single degree of freedom lumped-parameter models $[86,88]$ and numerical (mostly finite element) models $[56,57,89]$ have attracted the greatest attention. Although a harmonic input is predominantly considered, Luo et al. [90] proposed a model that can be used in applications where the excitation has a low frequency or has a discontinuous or variable amplitude, such as those from footsteps. Their model treats a ferroelectret as both a capacitor and a spring-mass-damper system that provides a restoring force when the applied compressive force is released (Fig. 17). The model includes both the voltage $V$ and the duration $t$ of an electrical signal generated by a ferroelectret, and relates them to the $d_{33}$ and the Young's modulus. This model enabled the calculation of the voltage, power output and energy output of energy harvesters based on cellular polymers. The output voltage of the ferroelectret is given by:

$$
V_{\text {out }}=\frac{F d_{33}(h-\Delta h)}{\varepsilon_{33} b l}
$$


Where $F$ is the compressive force, $b$ is the width of the specimen, $l$ is the length, $h$ is the thickness and $\Delta h$ is the change in thickness. It is of interest to note that the output voltage per unit stress, $\frac{V_{\text {out }}}{F / b l}$, it similar to the sensing figure of merit (Equation 20).

The generated electrical energy consists of the energy stored in the self-capacitor and that delivered to the electrical load (harvested), which is given by $\int \frac{V_{\text {out }}^{2}}{R} d t$. Based on this formulation, expressions were developed for the output energy and the duration $t$ of the signal.
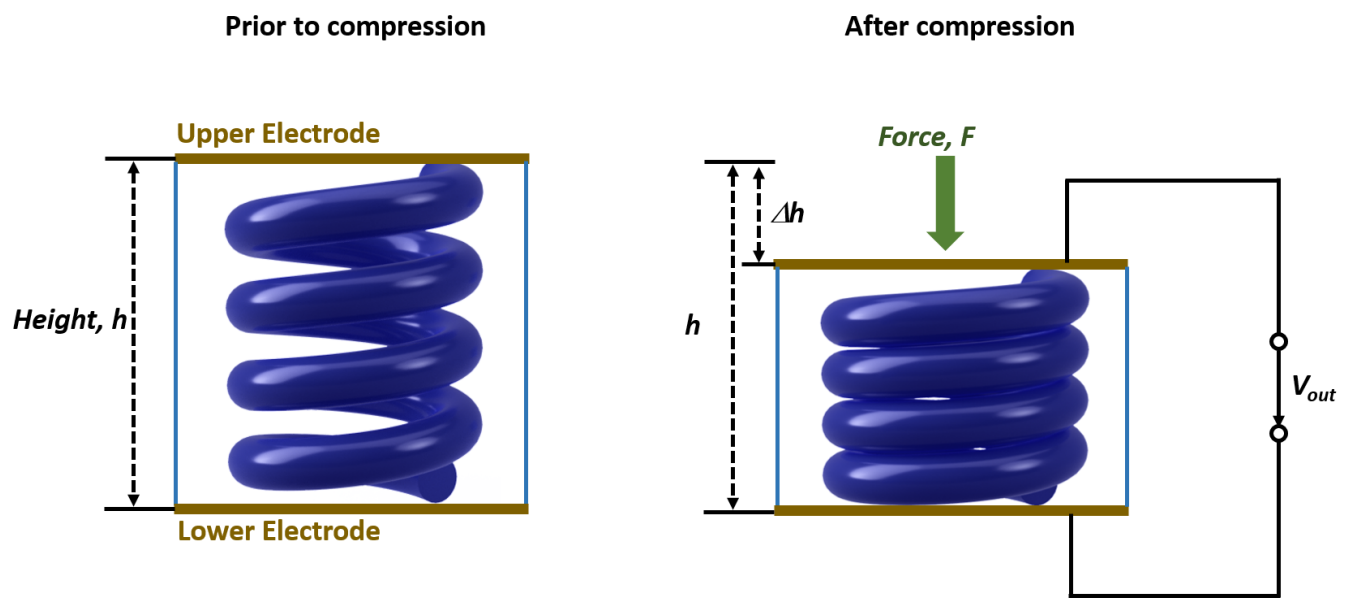

Fig. 17 A spring - mass - damper model of a ferroelectret, reproduced from [90]. (Copyright 2016 IOP Publishing)

Under the trapezoidal compressive force, the output voltage was obtained for samples measuring $60 \mathrm{~mm} \times 70 \mathrm{~mm}$ with a thickness of $70 \mu \mathrm{m}$. Loads are applied in the form of in-plane uniaxial and/or biaxial loading (Fig. 18A), as well as out-of-plane loading (Fig. 18B) which can be induced by direct force application on a stationary specimen or by inertial loading as in base-excited systems $[77,88]$. The terminals of the conductive electrodes covering the top and bottom surfaces are usually connected to resistive loads $[77,87]$ to estimate the power output. For the case of in-plane biaxial loading, the dynamic boundary strain components $S_{1}$ and $S_{2}$ induce a strain in the thickness direction $\left(S_{3}\right)$ due to the Poisson effect, which activates the ferroelectret material for power generation. The strain in the transverse direction is given by Anton et al [77] as: 


$$
S_{3}=-\frac{v_{13}+v_{23} v_{12}}{1-v_{12} v_{21}} S_{1}-\frac{v_{13} v_{21}+v_{23}}{1-v_{12} v_{21}} S_{2}
$$
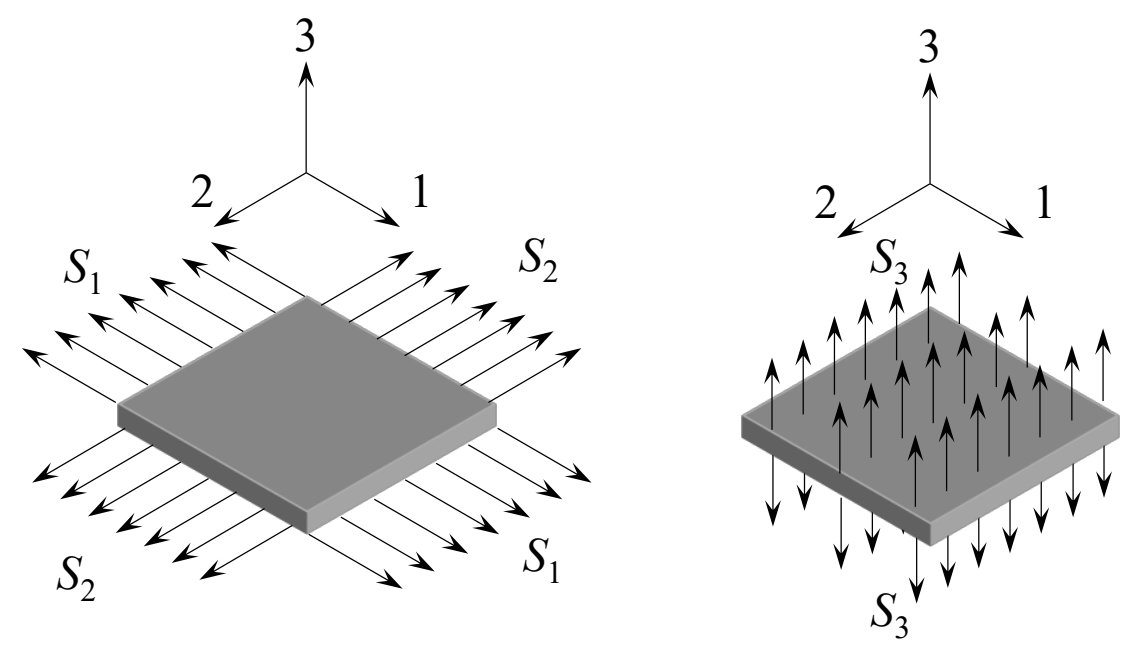

Fig. 18 Ferroelectret sample under in-plane, bi-axial loading (left) and out-of-plane loading (right). Partially redrawn from [77]. Copyright 2014 SAGE.

The electrical current flowing through the resistor is given by:

$$
\frac{d}{d t}\left(\int_{A} \vec{D} \cdot \vec{n} d A\right)=\frac{v}{R_{l}}
$$

Where the electric displacement $D_{3}$ is obtained from linear piezoelectricity as:

$$
D_{3}=d_{33} Y_{3}^{E} S_{3}+\varepsilon_{33}^{S} E_{3}
$$

Where $Y_{3}^{E}$ is the short-circuit elastic modulus in the thickness direction, $\varepsilon_{33}^{S}$ is the permittivity at constant strain and $E_{3}$ is the electric field which is assumed to be constant across the thickness. The dynamic equation that governs the voltage output across the resistor was hence obtained as:

$$
C_{p} \frac{d v}{d t}+\frac{v}{R_{l}}=\vartheta_{1} \frac{d S_{1}}{d t}+\vartheta_{2} \frac{d S_{2}}{d t}
$$

Where $C_{p}$ is the capacitance of the ferroelectret foam, while $\vartheta_{1}$ and $\vartheta_{2}$ are electromechanical coupling constants that depend on the electrode area, A. Accordingly, 
closed-form expressions for the output voltage per strain input and peak power were obtained.

For out-of-plane loading, Hillenbrand et al. [88] developed an analytical model for the power generated by an electret based energy harvester in response to an input acceleration by adapting the model of a single-layer material by Pomdrom et al. [81, 91, 92]. This was accomplished by replacing the piezoelectric constant $d_{33}$ in the harvester model with an equivalent piezoelectric constant $d_{33}$ in the electret harvester model. The generated power $P$ of a ferroelectret harvester can then be written as:

$$
P=\frac{m_{s}^{2} R_{l} d_{33, e q}^{2} \omega^{2} a^{2}}{\left[\left(\frac{\omega^{2}}{\omega_{0}^{2}}-1\right)^{2}+4 \zeta^{2} \frac{\omega^{2}}{\omega_{0}^{2}}\right]\left[1+\left(R_{l} C_{s} \omega\right)^{2}\right]}
$$

Where $R_{l}$ is the load resistance, $\omega_{0}$ is the resonance frequency of the harvester, $\omega$ is the circular frequency, and $\zeta$ is the damping ratio. The equivalent piezoelectric constant $d_{33}$, in the harvester model has to be defined and thus corresponds to the charge density (or charge $Q$ ) generated in short circuit by the transducer in response to a given applied stress $T$ (or force $F$ ). The restoring forces are due to the cellular ring, the mechanical springs, and the air gap. Assuming that the springs are much softer than the cellular ring, only this ring and the air gap have to be taken into consideration. A similar model was reported in $[4,6,11]$.

As an example, model predictions were compared by Pondrom et al. [80] with experimental data using harmonic excitation of a seismic mass (Fig. 19A) which could model harvesters with a range of ferroelectret layers and load resistances (Fig. 19B), with good agreement. 
(A)

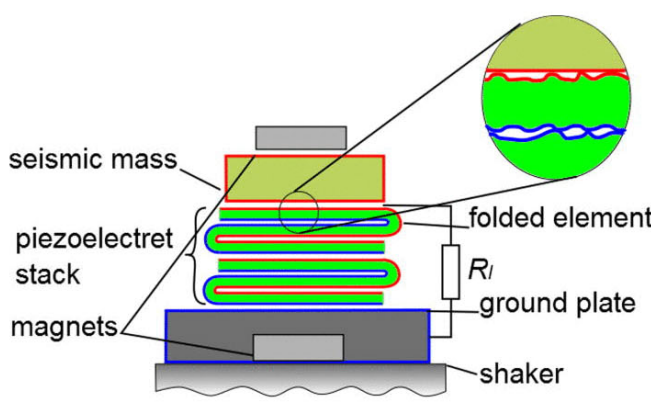

(B)

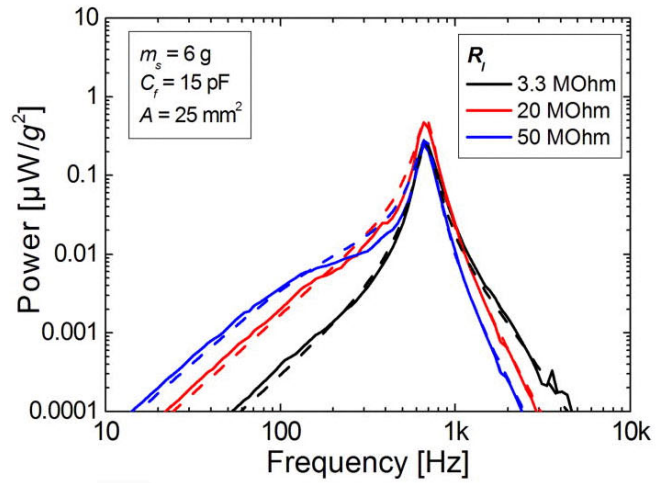

Fig. 19 (A) Experimental setup consisting of energy harvester and shaker. A ferroelectret stack of $s=2$ folded elements, each with $p=3$ layers, is shown. The stack is placed between the seismic mass and the ground plate and these are held together by two magnets. The resistor $R_{l}$ allows measurement of electrical power generated [80]. (B) Power generated by energy harvesters with various numbers of layers. Full lines represent data from the measurements, the dashed lines show the simulation results. The 10 layer stack consists of films connected in series $(s=10, p=1)$, and the 9 layer stack of 3 films, each folded twice, and connected in series $(p=3, s=3)[80]$. Reprinted with permission of AIP Publishing.

Ray et al. [93] examined two 20-layer foam stacks (Stack 1 and Stack 2) and a 40-layer stack (Stack 3; composed of Stack 1 and Stack 2 connected mechanically in series and electrically in parallel) to achieve a more practical optimal load resistance. Using the fundamental equations of piezoelectricity, Ennawaoui et al.[87] proposed a theoretical model of the power density that harvested using the ferroelectret polymers, as shown below,

$$
P=2 \pi^{2} R_{1} \frac{\left(\sigma_{G}\left[\frac{R(1+V)}{(R+V)^{2}} f A S_{M}\right]\right)^{2}}{1+4 \pi^{2}\left(\varepsilon_{33}-\sigma_{G}^{2}\left[\frac{R(1+V)}{(R+V)^{2}}\right]^{2}\right)^{2}\left(\frac{R_{1}}{e} f A\right)^{2}}
$$

where $\mathrm{R}_{1}$ is the resistor through which power is dissipated. $V=\frac{X_{G}}{X_{P}}$ where, $X_{G}$ and $X_{P}$ are the thicknesses of the void and the polymer, respectively. $R=\frac{\varepsilon_{G}}{\varepsilon_{P}}$ where, $\varepsilon_{G}$ and $\varepsilon_{P}$ are the relative permittivity (dielectric constant) of the void and the polymer, respectively. $A$ is the active surface of the polymer, $\sigma_{G}$ is the charge density, $S_{M}$ is the strain and $f$ is the resonance frequency. Thus, the theoretical model allows determination of the power density based on cell porosity, thickness, and dielectric constant. 
The model shows that power density increases with increasing porosity level, up to a critical limit after which the power density decreases. Beyond such a limit, the pores coalesce and there is a resulting loss of the local dipoles that provide piezoelectricity. Gross et al. proposed a micromechanical modeling to investigate the charging and discharging behaviour of a soft ferroelectret film and demonstrated via $2 \mathrm{D}$ finite element simulations that electric breakdown within lens shaped voids provides a piezoelectric response that is dependent on void size [94]. In addition, Shi et al.[56] provided an analytical result via ANSYS simulation considering previously proposed analytical models which numerically illustrates the impact of the different void geometry for a PDMS based ferroelectret. When the ratio of the effective area of the solid and void layers is increased, the piezoelectricity of PDMS ferrelectret was reduced, which indicates that the piezoelectric properties of the PDMS ferroelectret improved with increasing void area since the polarization of the voids are responsible for the piezoelectric properties. Fig. 20A shows a comparison of ANSYS simulation and analytical model results to illustrate the influence of the surface charge density based on the designed geometry. The theoretical maximum piezoelectric coefficient was $d_{33}$ $\sim 220 \mathrm{pC} / \mathrm{N}$; the experimental maximum was $d_{33} \sim 172 \mathrm{pC} / \mathrm{N}$. Later, they have used the PDMS ferroelectret as energy harvester which generates $0.12 \mu \mathrm{W} / \mathrm{cm}^{2}$ power density under a square compressive force of $800 \mathrm{~N}$ at $17 \mathrm{~Hz}$; as shown in Fig. 20B [89] .

(A)

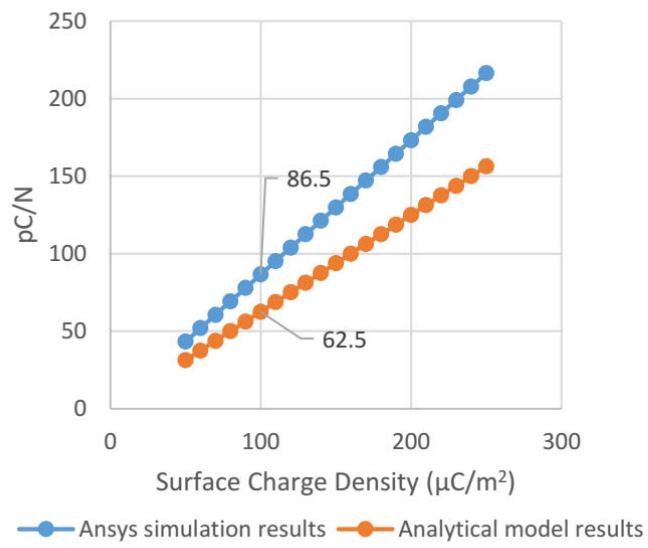

(B)

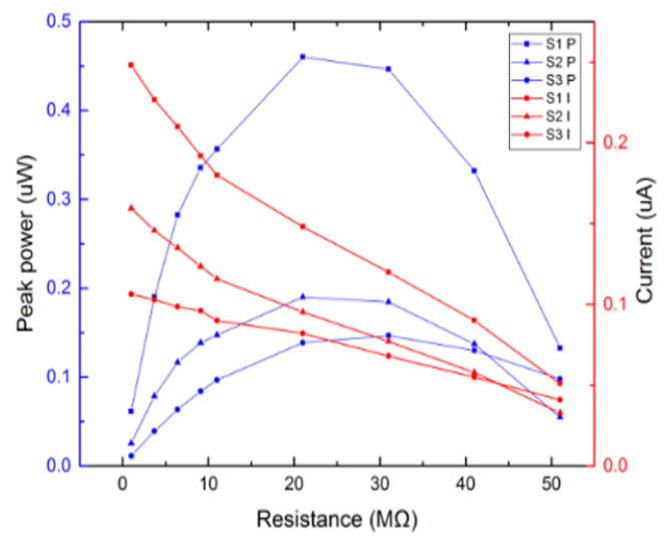

Fig. 20 (A) analytical model results and ANSYS simulated $d_{33}$ results for rectangle voids [56], (B) experimentally harvested power under $800 \mathrm{~N}$ compressive force and $17 \mathrm{~Hz}$ force frequency [89].

Creative Commons Attribution 3.0 licence. 
Several modelling approaches have been proposed for ferroelectret based sensor applications, which could be applicable to energy harvesting applications. Kachroudi et al.[60] proposed an equivalent electrical circuit model and explained how it can be used to predict the micro-structured PDMS complex permittivity versus frequency and temperature. Kärki et al.[86] developed a lumped-parameter model for sensor operation of piezoelectric and ferroelectret based polymer materials that describes the interaction between a mechanical motion and an electrical signal. Luo et al.[90] proposed a theoretical model that can be used to predict the energy output of a ferroelectret by knowing its parameters, and therefore optimize the harvester design for specific energy harvesting application. Finally, Rupitsch et al.[35] studied a finite element based modelling approach, in particular, a microscopic as well as a macroscopic model of the cellular structured ferroelectret materials, namely the socalled EMFi (Electro-Mechanical-Film) material. These approaches could be adapted to energy harvesting applications and optimisation of performance.

In summary, a variety of models exists were the macro-scale properties of the ferroelectret (such as permittivity and $d_{33}$ ) are inserted in the fundamental equations of piezoelectric materials. There remains scope for more micro-scale modeling to understand pore geometry and conditions for polarization and resulting piezoelectric properties, in particular optimization of figures of merit for energy harvesting applications, such as Eqn. 1. For energy harvesting applications there is less on continuous system modeling, with a focus primarily on lumped parameter models.

\section{Energy harvesting applications}

A number of energy harvesting demonstrators based on ferroelectrets have been reported. These include cantilever based systems [43], nanogenerator based devices [33, 95], wearable electronics [69], and ferroelectrets combined with other nanogenerators [96]. Table 1 outlines the power generated for the range of devices, which range from low powers of $2.5 \mathrm{nW}$ to higher levels of $3.5 \mathrm{~mW}$.

Zhang et al.[4] demonstrated energy harvesting applications of a ferroelectret nanogenerator (FENG) based on ferroelectret fluorocarbon polymers that have a large 
low-frequency transverse piezoelectric coefficient, for example $g_{31}=3.0 \mathrm{Vm} / \mathrm{N}$. The design of the experimental setup, as shown in Fig. 21A, consisted of a harvester and shaker where the ferroelectret film was fixed at both ends on the supporting structure. The system had a slight static deflection due to the seismic mass, and was also dynamically deflected by the seismic mass in response to acceleration from an electrodynamic shaker. Images for the ferroelectret harvester with different seismic masses are shown in Fig. 21B. The harvested power was $57 \mu \mathrm{W}$ and $109 \mu \mathrm{W}$ for seismic masses of $0.09 \mathrm{~g}$ and $0.3 \mathrm{~g}$, respectively. To demonstrate the potential application of the harvested power, the harvester was combined with a rectifier bridge to condition the AC current and power a light-emitting diode (LED), as shown in the left side of Fig. 20B. The experimental setup is shown in the right-side part of Fig. 21C, where an acceleration of $0.3 \mathrm{~g}$ was sufficient to power the LED.

(A)

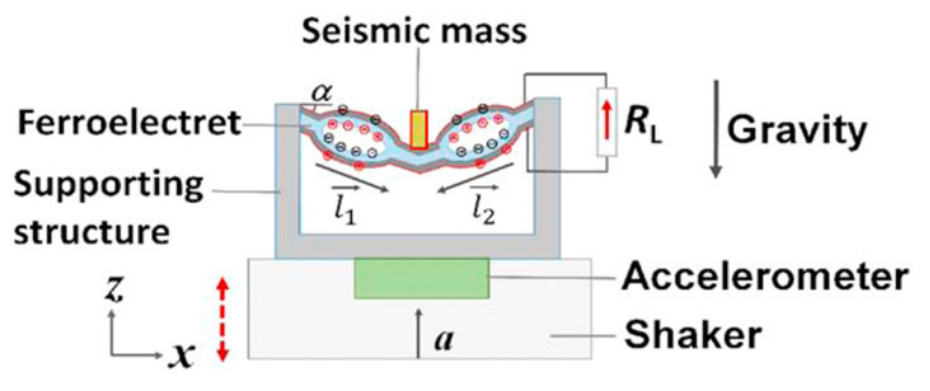

(B)

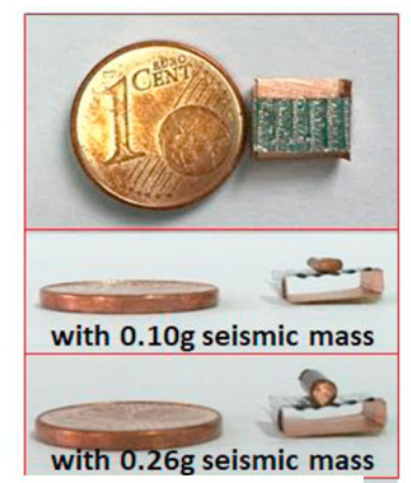

(C)
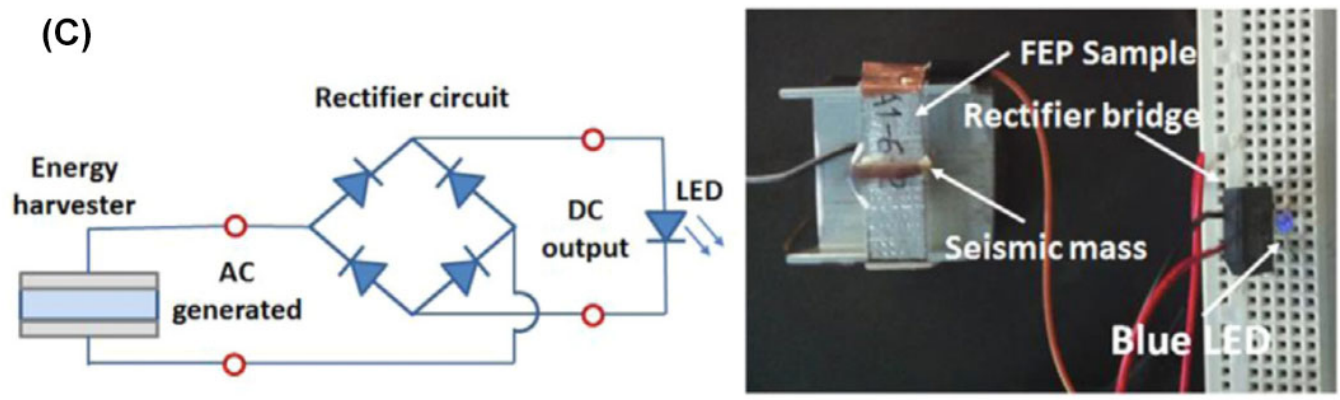

Fig. 21 (A) Schematic of experimental setup consisting of ferroelectret harvester and shaker, (B)

Photographs of the harvester, (C) setup of energy harvester to power LED [4]. Reprinted with permission from Elsevier 2018.

The capability of a ferroelectret based harvester that consisted of a stack of seven polypropylene ferroelectret (PPFE) film layers was demonstrated by Li et al. who converted human motion into electrical energy[95]. They showed that by pressing 
with a hand, a ferroelectret harvester connected with a Schottky bridge rectification circuit (Fig. 22A) could light 20 LEDs (Fig. 22B). Using this concept, they developed a self-powered liquid crystal display (LCD) by integrating the harvester with a 4-bit LCD screen. When the user tapped the self-powered LCD screen, the word 'PLAY' was displayed on the screen; this system did not employ rectifiers or charging circuits (Fig. 22C). Clearly stacking provides an attractive route to improve the harvested power. Another promising application is demonstrating human-machine interfacing and information exchange by a self-powered keyboard. The structural design and image of the thin film keyboard are shown in Fig. 22D and E, respectively. The developed ferroelectret-based keyboard could be rolled (Fig. 22F) and folded (Fig. 22G) while maintaining its functionality.

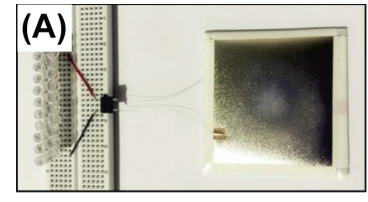

(B)
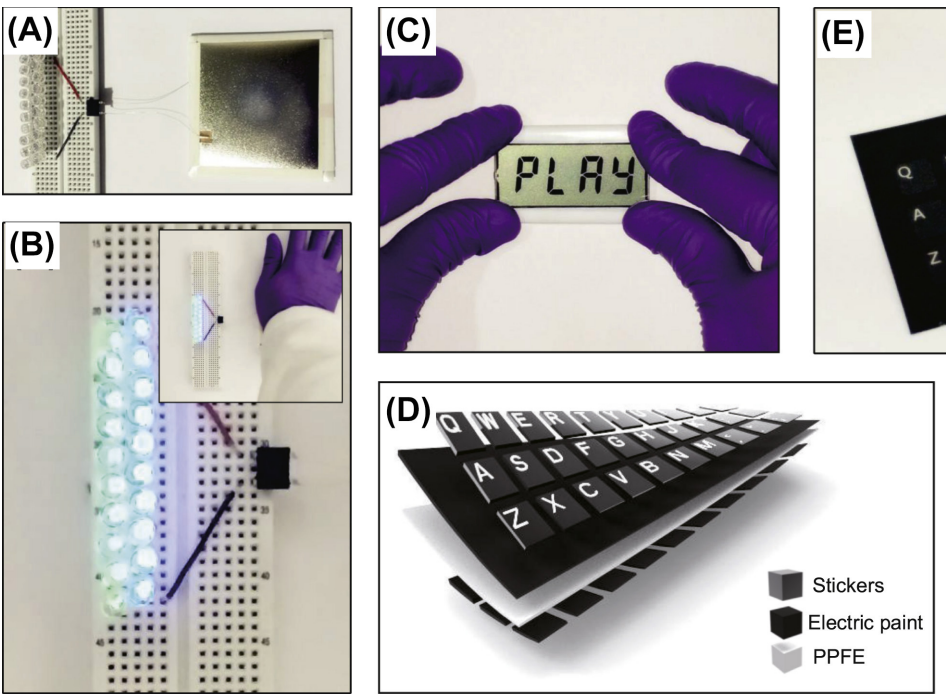
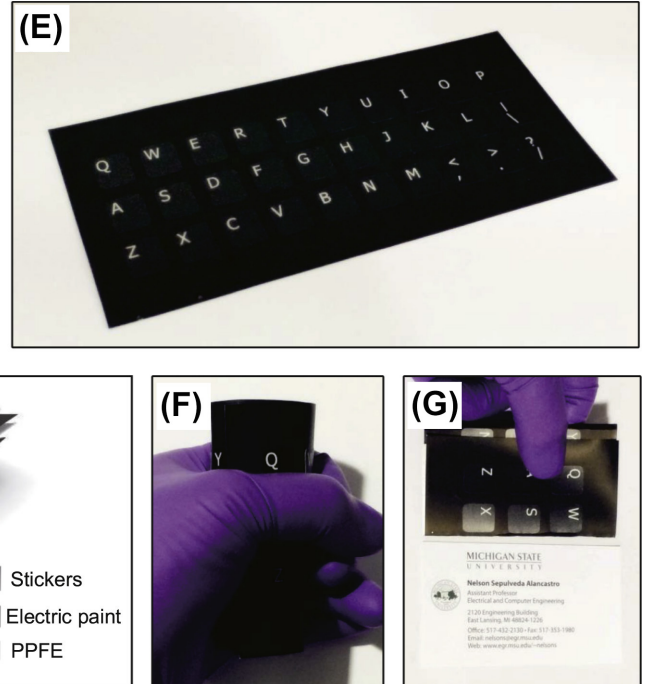

Fig. 22 Applications of polypropylene ferroelectret nanogenerator (FENG) for versatile mechanical energy harvesting [95]. Reprinted with permission from Elsevier 2016.

In order to demonstrate the applicability of the ferroelectret harvesters in a harsh environment, Zhong et al.[33] fabricated PET/EVA/PET laminated films and charged them using a corona charging at a high voltage of up to $-20 \mathrm{kV}$ (Fig. 23A). The $d_{33}$ coefficient of the developed generator reached $\sim 6300 \mathrm{pC} / \mathrm{N}$, which was sustained for several weeks (observed up to 6 weeks) when operated in an indoor atmosphere (Fig. 23B). The generator could operate six blue LEDs connected in series by hand pressing when soaked in water (Fig. 23C). 


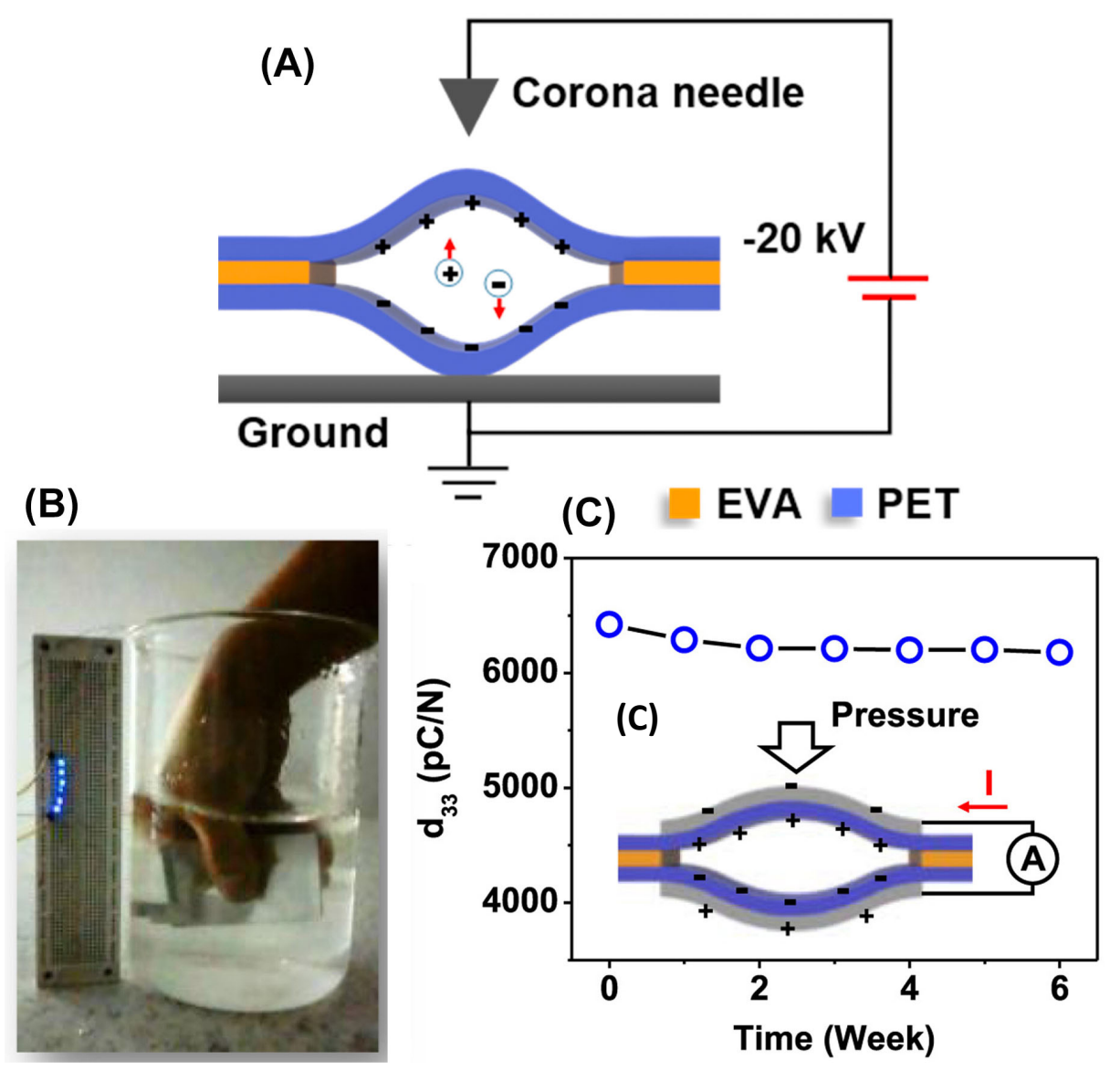

Fig. 23 (A) Ferroelectret structure, (B) LEDs lit up by hand pressing the ferroelectret generator under water (C) Stability of piezoelectric coefficient with time [33]. Reprinted with permission from Elsevier 2017.

In addition, the operation of a ferroelectret harvester for wireless signal transmission was demonstrated by Luo et al.[97] by fabricating a flexible energy harvesting insole made of 80-layers of porous polypropylene (PP) ferroelectrets (Fig. 24A). Insole samples with 10-layer, 20-layer, 30-layer, 40-layer and 80-layer of PP ferroelectrets were evaluated. During walking, electrical pulses generated from the ferroelectret insole are rectified and used to charge a $2.2 \mu \mathrm{F}$ capacitor. It was shown that for every single footstep, the 10, 20, 30, 40 and 80-layer ferroelectret insoles charged $19.8 \mu \mathrm{J}$, $31.9 \mu \mathrm{J}, 40.2 \mu \mathrm{J}, 65.6 \mu \mathrm{J}$ and $100.9 \mu \mathrm{J}$ of energy, respectively, into a capacitor (Fig. 24B). Fig. 24A shows the ferroelectret insole connected to a power conditioning circuit and a ZigBee transmitter. Wireless transmission was monitored by a receiver placed 8 meters from the transmitter and an LED on the receiver was turned off when an 8-bit data was received. During walking on the ferroelectret insole, the LED light turned off 
for every 3 to 4 footsteps, indicating a wireless transmission has been powered by the insole.
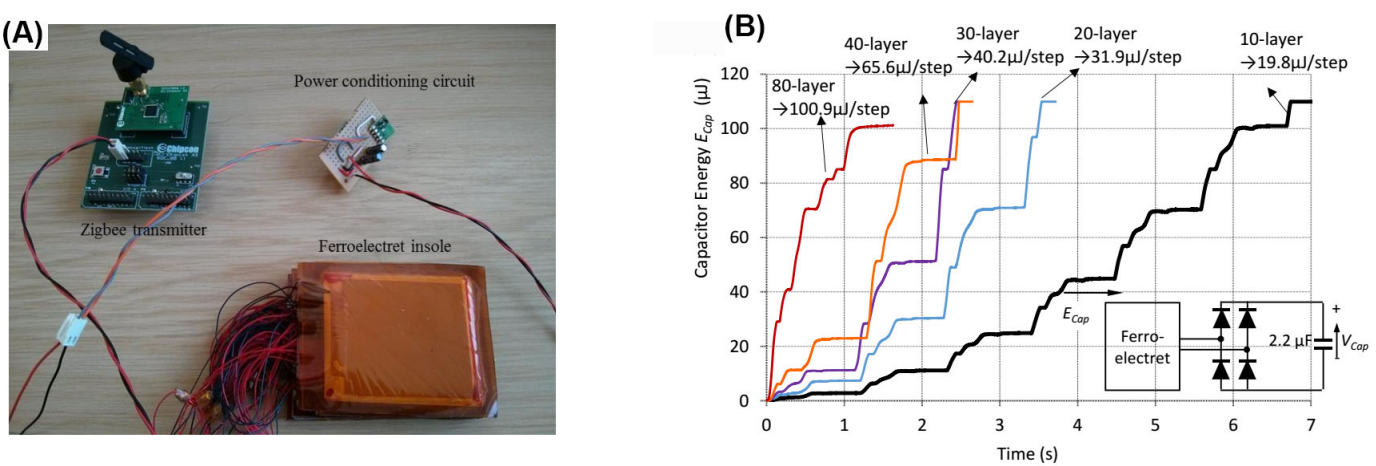

Fig. 24 (A) Ferroelectret insole connecting to the power conditioning circuit and the ZigBee transmitter. (B) energy charging curve of a $2.2 \mu \mathrm{F}$ capacitor charged by 10, 20, 30, 40 and 80 layers of ferroelectrets. [97] Creative Commons Attribution 3.0 licence.

Kachroudi et al. [43] examined air-spaced cantilevers (Fig. 25A) manufactured from a PDMS ferroelectret material (Fig. 25B) for energy harvesting. To study the energy harvested from mechanical vibrations, the ferroelectret was connected to variable resistors in parallel with an instrumentation amplifier. The system was excited with an acceleration of $0.1 \mathrm{~g}$ at frequencies ranging from $3.7 \mathrm{~Hz}$ to $200 \mathrm{~Hz}$. The resistance varied from 130.2 $\mathrm{M} \Omega$ to $295.3 \mathrm{M} \Omega$. An optimum power was found for a resistance of $217 \mathrm{M} \Omega$ and for a capacitance of the material of $171 \mathrm{pF}$; and such impedance matching of the resistive electric load to the capacitive impedance of the generator is common in ferroelectric systems. The generated power reached a maximum value of $136 \mathrm{nW}$ and has a constant value of $103 \mathrm{nW}$ for frequencies ranging from $5 \mathrm{~Hz}$ to $200 \mathrm{~Hz}$.

(A)

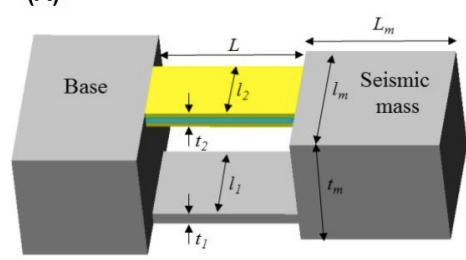

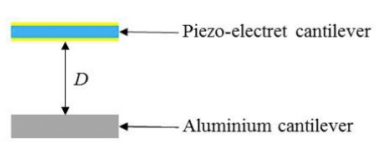

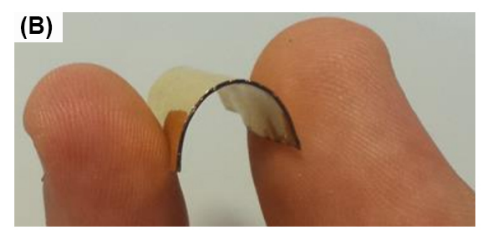

Fig. 25 (A) Schematic structures of the air-spaced cantilevers and (B) image of the obtained flexible piezo-electret material [43]. Creative Commons Attribution 3.0 licence. 
With regard to candidate material, porous polymers with highly dielectric and electrically insulating characteristics and high permittivity are promising ferroelectret materials. In addition, polymers with high permittivity, such as PVDF, can act to increase the permittivity contrast between the polymer and pore space and concentrates the electric field in the pore space due to Gauss's law $\left(E_{f}=q / A \cdot \varepsilon\right.$, where $q$ is charge, $A$ is area, $\varepsilon$ is permittivity of the polymer, and $E_{f}$ is electric field). This field concentration facilitates Townsend's breakdown [39] to create the ferroelectret. Moreover, for the optimised structure for energy harvesting applications, although the generated power from ferroelectrets with a single pore layer can be as high as 73 $\mu \mathrm{W}$ [98], it still can be improved to around milli-watt (e.g. $0.641 \mathrm{~mW}$ [68], $1 \mathrm{~mW}$ [80]) via simple approaches such as stacking or folding many single pore layers together to make a complete device, as in Figs. 21 and 27. This is particularly attractive for energy harvesting since the ability to increase overall area of the ferroelectret increases the total amount of charge (and therefore current) to be harvested. For sensing the output voltage per unit stress is independent of area, see Equation 14.

While emphasis in this review was placed on providing an overview of ferroelectrets that have been considered for energy harvesting, it is known that ferroelectrets also possess unique characteristics that favour their use in acoustic and other pressure sensing. While the figure of merit for energy harvesting is given by Equation 1, for sensing applications there is a need to maximise the voltage per unit stress and for piezoelectric materials this is related to:

$$
\text { FoM } M_{\text {sensor }}=d_{i j} /_{\varepsilon_{33}^{X}}=g_{i j}
$$

where $g_{i j}$ is a measure of the electric field per unit stress $\left(g_{33}\right.$ or $\left.g_{31}\right)$. This also indicates the need for high piezoelectric $d_{i j}$ coefficients and low permittivity and materials that exhibit high acoustic or high pressure sensitivity may also be of interest for energy harvesting such as the work of Carpi [21], Bovtun [30], Strobel [99], and Paajanen [34]. 
As an example of current state-of-the art of ferroelectret sensing, Wang et al [100] presented a ferroelectret pressure sensor with very high $d_{33} \sim 7380 \mathrm{pC} / \mathrm{N}$ based on a fluorocarbon fabricated by a three-stage hot pressing method along with a detailed comparison with existing ferroelectret based sensors. While the material has not been evaluated for harvesting, the high $d_{33}$ and inherently low permittivity of the porous polymer make it an interesting candidate for harvesting; see Equation 1. For further reading on the acoustic applications of electrets/ferroelectrets, the reader is referred to the reference by Carpi [21].

\section{Conclusions and perspective}

Ferroelectrets have gained significant interest for mechanical energy harvesting applications due to their excellent piezoelectric response because of the highly compliant nature of porous polymers, which leads to a large deformation under mechanical loads. As they show much smaller pyroelectric coefficients, their application in thermal energy harvesting has not attracted much interest. This review has covered the progress to date on the materials, manufacturing methods, key microstructural features, performance, modelling and devices that make use of this new class of materials in mechanical energy harvesting applications. Table 1 provides a summary of the polymer systems, fabrication approaches, pore characteristics, dielectric and piezoelectric properties as well as generated power levels.

A range of thermoplastic polymers, including ferroelectric polymers, have been used to fabricate ferroelectrets. Bulk polarisation is induced by corona poling resulting in micro barrier plasma discharge within the pores. Nano-particle polymer ferroelectret composites have been shown to have self-poling features as well as superior piezoelectric properties as a result of the coupling between intrinsic ferroelectric polymer with the trapped charges in the porous ferroelectric membranes. Polyelectrolyte filled polymer ferroelectret composites also show enhanced piezoelectric response due to a combination of several polarisation mechanisms. It is important to note that often high losses and high permittivity values are associated with the presence of electrically conductive third phase. 
Manufacturing methods include foaming processes, stretching particulate filled polymers, hot pressing of sandwich layer structures that a porous ferroelectret is clamped between two dense polymer layers for improved charge density and finally micro-patterning using grooved templates. The porosity size, morphology and orientation with respect to the poling direction affect the gaseous phase breakdown voltage as well as materials compliance and effective charging area within the pores, thus affecting the piezoelectric properties of ferroelectrets. A wide distribution of pore size results in a tilting of the polarisation-electric field loop. The size of the voids is varied by the manufacturing conditions such as polymer viscosity, composition, pressure and temperature and can be adjusted by stretching or compression post fabrication. Ideal pore size to facilitate Townsend discharge to create polarised porous structure varies between $10-100 \mu \mathrm{m}$ and the aspect ratio above four are favourable.

Analytical modelling approaches are reviewed which examine the effect of microstructural features on the electro-mechanical performance of the ferroelectrets subjected to a mechanical excitation. The polymer ferroelectret is considered as a composite consisting of a gaseous phase dispersed in a solid polymer matrix. Anisotropy of the lens-like pores and the low symmetry of the charge distribution, results in high piezoelectric charge coefficient in the thickness direction which is typically two orders of magnitude larger than the in-plane piezoelectric coefficients. It has been shown that there is a critical pore fraction correlated with maximum generated power density, beyond which the pores coalesce, and piezoelectric response diminishes. There is further scope for micro-scale modelling to understand pore geometry and conditions for polarisation and resulting piezoelectric properties.

Ferroelectrets have been used to develop mechanical energy harvesting devices and nanogenerators for wearable electronics, self-powered screens without the use of rectifiers, foldable flexible keyboards and wireless signal transmission devices. Cantilever based systems made of ferroelectrets have also been shown to effectively generate energy from mechanical vibrations. A number of new applications in high strain environments to exploit highly compliant ferroelectret energy harvesters could 
be explored, along with folding and stacking to improve power levels. There is also a growing need for such ideas to be transferred into commercial activities to fully use the potential of this class of materials for a wide range of energy harvesting applications. To extend the application spectrum further, ferroelectret polymers with improved pyroelectric properties are desirable. Although the low thermal sensitivity of ferroelectrets makes them poor thermal sensors or harvesters, it is beneficial when used for the thermally stable electromechanical applications. However, new approaches in materials characteristic can be explored to improve the thermal sensitivity of ferroelectrets comparable to their ceramic ferroelectric counterparts to also make them suitable for pyroelectric energy harvesting. Ferroelectret materials are appealing because of their benign nature and cost effectiveness. The achievement of greater figures of merit remains to be a key challenge that would enable their application in wider energy harvesting applications. One viable approach to tackle this challenge is through the development of novel fabrication techniques that would result in more uniform and well-controlled geometry of cellular voids to achieve ideal cellular structures.

\section{References}

[1] S. Bauer, Piezo-, pyro- and ferroelectrets: soft transducer materials for electromechanical energy conversion, IEEE Transactions on Dielectrics and Electrical Insulation 13(5) (2006) 953-962. 
[2] R.G.-M. Siegfried Bauer, and Gerhard M. Sessler, Ferroelectrets: Soft Electroactive Foams for Transducers, Physics Today 57(2) (2004) 37.

[3] X. Zhang, G.M. Sessler, Y. Wang, Fluoroethylenepropylene ferroelectret films with cross-tunnel structure for piezoelectric transducers and micro energy harvesters, Journal of applied physics 116(7) (2014) 074109.

[4] X. Zhang, P. Pondrom, G.M. Sessler, X. Ma, Ferroelectret nanogenerator with large transverse piezoelectric activity, Nano Energy 50 (2018) 52-61.

[5] S. Junjie, Y. Sheng, B. Steve, An easy to assemble ferroelectret for human body energy harvesting, Smart Materials and Structures 27(8) (2018) 084005.

[6] Y. Wang, L. Wu, X. Zhang, Energy harvesting from vibration using flexible floroethylenepropylene piezoelectret films with cross-tunnel structure, IEEE Transactions on Dielectrics and Electrical Insulation 22(3) (2015) 1349-1354.

[7] J. Briscoe, S. Dunn, Piezoelectric nanogenerators - a review of nanostructured piezoelectric energy harvesters, Nano Energy 14 (2015) 15-29.

[8] Z.L. Wang, T. Jiang, L. Xu, Toward the blue energy dream by triboelectric nanogenerator networks, Nano Energy 39 (2017) 9-23.

[9] H. You, X. Ma, Z. Wu, L. Fei, X. Chen, J. Yang, Y. Liu, Y. Jia, H. Li, F. Wang, H. Huang, Piezoelectrically/pyroelectrically-driven vibration/cold-hot energy harvesting for mechano-/pyro- bi-catalytic dye decomposition of NaNbO3 nanofibers, Nano Energy 52 (2018) 351-359.

[10] M.Q. Le, J.-F. Capsal, M. Lallart, Y. Hebrard, A. Van Der Ham, N. Reffe, L. Geynet, P.-J. Cottinet, Review on energy harvesting for structural health monitoring in aeronautical applications, Progress in Aerospace Sciences 79 (2015) 147-157.

[11] X. Zhang, L. Wu, G.M. Sessler, Energy harvesting from vibration with cross-linked polypropylene piezoelectrets, AIP Advances 5(7) (2015) 077185.

[12] A.C. Lopes, C.M. Costa, C.J. Tavares, I.C. Neves, S. Lanceros-Mendez, Nucleation of the Electroactive $\gamma$ Phase and Enhancement of the Optical Transparency in Low Filler Content Poly(vinylidene)/Clay Nanocomposites, The Journal of Physical Chemistry C 115(37) (2011) 18076-18082.

[13] M.-G. Kang, W.-S. Jung, C.-Y. Kang, S.-J. Yoon, Recent Progress on PZT Based Piezoelectric Energy Harvesting Technologies, Actuators 5(1) (2016) 5. 
[14] H. Kim, S. Priya, H. Stephanou, K. Uchino, Consideration of Impedance Matching Techniques for Efficient Piezoelectric Energy Harvesting, IEEE Transactions on Ultrasonics, Ferroelectrics, and Frequency Control 54(9) (2007) 1851-1859.

[15] D.B. Deutz, J.-A. Pascoe, B. Schelen, S. van der Zwaag, D.M. de Leeuw, P. Groen, Analysis and experimental validation of the figure of merit for piezoelectric energy harvesters, Materials Horizons 5(3) (2018) 444-453.

[16] H. Kim, Y. Tadesse, S. Priya, Piezoelectric Energy Harvesting, in: S. Priya, D.J. Inman (Eds.), Energy Harvesting Technologies, Springer US, Boston, MA, 2009, pp. 3-39.

[17] G.S. Neugschwandtner, R. Schwödiauer, S. Bauer-Gogonea, S. Bauer, M. Paajanen, J. Lekkala, Piezo- and pyroelectricity of a polymer-foam space-charge electret, Journal of Applied Physics 89(8) (2001) 4503-4511.

[18] X. Qiu, Patterned piezo-, pyro-, and ferroelectricity of poled polymer electrets, Journal of Applied Physics 108(1) (2010) 011101.

[19] H.H.S. Chang, R.W. Whatmore, Z. Huang, Pyroelectric effect enhancement in laminate composites under short circuit condition, Journal of Applied Physics 106(11) (2009) 114110.

[20] F. Carpi, Electromechanically Active Polymers, Polymer International 59(3) (2010) 277-278.

[21] F. Carpi, Electromechanically Active Polymers, Springer International Publishing Switzerland, Springer International Publishing Switzerland 2016, 2016.

[22] A. Mohebbi, F. Mighri, A. Ajji, D. Rodrigue, Cellular Polymer Ferroelectret: A Review on Their Development and Their Piezoelectric Properties, Advances in Polymer Technology 37(2) (2018) 468-483.

[23] R. Gerhard-Multhaupt, Less can be more. Holes in polymers lead to a new paradigm of piezoelectric materials for electret transducers, IEEE Transactions on Dielectrics and Electrical Insulation 9(5) (2002) 850-859.

[24] M. Lindner, H. Hoislbauer, R. Schwodiauer, S. Bauer-Gogonea, S. Bauer, Charged cellular polymers with "ferroelectretic" behavior, IEEE Transactions on Dielectrics and Electrical Insulation 11(2) (2004) 255-263.

[25] Y.-P. Wan, Z. Zhong, Effective electromechanical properties of cellular piezoelectret: A review, Acta Mechanica Sinica 28(4) (2012) 951-959. 
[26] M. Wegener, S. Bauer, Microstorms in Cellular Polymers: A Route to Soft Piezoelectric Transducer Materials with Engineered Macroscopic Dipoles, ChemPhysChem 6(6) (2005) 1014-1025.

[27] Y. Suzuki, Electrostatic/Electret-Based Harvesters, in: E.Y.a.S.R. D. Briand (Ed.), Micro Energy Harvesting2015.

[28] G.M. Sessler, J.E. West, Electret transducers: a review, The Journal of the Acoustical Society of America 53(6) (1973) 1589-1600.

[29] S. Bauer, S. Bauer-Gogonea, F. Camacho-Gonzales, M. Dansachmuller, I. Graz, M. Kaltenbrunner, J.G. Leonhartsberger, R. Schwodiauer, Ferroelectrets: polymer-foam space-charge electrets with ferroelectric-like behaviour, 2005 12th International Symposium on Electrets, 2005, pp. 24-27.

[30] V. Bovtun, J. Döring, J. Bartusch, U. Beck, A. Erhard, Y. Yakymenko, Ferroelectret non-contact ultrasonic transducers, Applied Physics A 88(4) (2007) 737-743.

[31] B. Wang, C. Liu, Y. Xiao, J. Zhong, W. Li, Y. Cheng, B. Hu, L. Huang, J. Zhou, Ultrasensitive cellular fluorocarbon piezoelectret pressure sensor for self-powered human physiological monitoring, Nano Energy 32 (2017) 42-49.

[32] L. Jin, W. Deng, Y. Su, Z. Xu, H. Meng, B. Wang, H. Zhang, B. Zhang, L. Zhang, X. Xiao, M. Zhu, W. Yang, Self-powered wireless smart sensor based on maglev porous nanogenerator for train monitoring system, Nano Energy 38 (2017) 185-192.

[33] J. Zhong, Q. Zhong, X. Zang, N. Wu, W. Li, Y. Chu, L. Lin, Flexible PET/EVA-based piezoelectret generator for energy harvesting in harsh environments, Nano Energy 37 (2017) 268-274.

[34] M. Paajanen, J. Lekkala, K. Kirjavainen, ElectroMechanical Film (EMFi) - a new multipurpose electret material, Sensors and Actuators A: Physical 84(1) (2000) 95-102. [35] S.J. Rupitsch, R. Lerch, J. Strobel, A. Streicher, Ultrasound transducers based on ferroelectret materials, IEEE Transactions on Dielectrics and Electrical Insulation 18(1) (2011) 69-80.

[36] T. Xu, C.A. Wang, Control of pore size and wall thickness of 3-1 type porous PZT ceramics during freeze-casting process, Materials \& Design 91 (2016) 242-247. 
[37] D.R. Xunlin Qiu, and Werner Wirges, Properties and Applications of Ferroelectrets, in: M.V.d.V.M.W.H.J. Fecht (Ed.), The Nano-Micro Interface: Bridging the Micro and Nano Worlds2015.

[38] C. Wan, C.R. Bowen, Multiscale-structuring of polyvinylidene fluoride for energy harvesting: the impact of molecular-, micro- and macro-structure, Journal of Materials Chemistry A 5(7) (2017) 3091-3128.

[39] R.W. Crowe, J.K. Bragg, V.G. Thomas, Space Charge Formation and the Townsend Mechanism of Spark Breakdown in Gases, Physical Review 96(1) (1954) 10-14.

[40] M. Wegener, W. Wirges, R. Gerhard-Multhaupt, Piezoelectric Polyethylene Terephthalate (PETP) Foams - Specifically Designed and Prepared Ferroelectret Films, Advanced Engineering Materials 7(12) (2005) 1128-1131.

[41] P.A. Tipler, College Physics, Worth Publishers Inc.,U.S. 1987.

[42] J. Zhong, Q. Zhong, G. Chen, B. Hu, S. Zhao, X. Li, N. Wu, W. Li, H. Yu, J. Zhou, Surface charge self-recovering electret film for wearable energy conversion in a harsh environment, Energy \& Environmental Science 9(10) (2016) 3085-3091.

[43] A. Kachroudi, S. Basrour, L. Rufer, F. Jomni, Air-spaced PDMS piezo-electret cantilevers for vibration energy harvesting, Journal of Physics: Conference Series, IOP Publishing, 2016, p. 012072.

[44] C. Livermore, Voldman, Joel, IMIT OpenCourseWare, 6.777J / 2.372J Design and Fabrication of Microelectromechanical Devices, 2007.

[45] B. Notario, J. Pinto, M.A. Rodríguez-Pérez, Towards a new generation of polymeric foams: PMMA nanocellular foams with enhanced physical properties, Polymer 63 (2015) 116-126.

[46] L.J.M. Jacobs, M.F. Kemmere, J.T.F. Keurentjes, Sustainable polymer foaming using high pressure carbon dioxide: a review on fundamentals, processes and applications, Green Chemistry 10(7) (2008) 731-738.

[47] L. Urbanczyk, C. Calberg, C. Detrembleur, C. Jérôme, M. Alexandre, Batch foaming of SAN/clay nanocomposites with ScCO2: A very tunable way of controlling the cellular morphology, Polymer 51(15) (2010) 3520-3531. 
[48] B.-X. Xu, H.v. Seggern, S. Zhukov, D. Gross, Continuum modeling of charging process and piezoelectricity of ferroelectrets, Journal of Applied Physics 114(9) (2013) 094103.

[49] P. Fang, X. Qiu, W. Wirges, R. Gerhard, L. Zirkel, Polyethylene-naphthalate (PEN) ferroelectrets: cellular structure, piezoelectricity and thermal stability, IEEE Transactions on Dielectrics and Electrical Insulation 17(4) (2010) 1079-1087.

[50] A. Mohebbi, F. Mighri, A. Ajji, D. Rodrigue, Polymer ferroelectret based on polypropylene foam: Piezoelectric properties improvement using post-processing thermomechanical treatment, Journal of Applied Polymer Science 134(10) (2017).

[51] N. Wu, X. Cheng, Q. Zhong, J. Zhong, W. Li, B. Wang, B. Hu, J. Zhou, Cellular Polypropylene Piezoelectret for Human Body Energy Harvesting and Health Monitoring, Advanced Functional Materials 25(30) (2015) 4788-4794.

[52] M. Sauceau, J. Fages, A. Common, C. Nikitine, E. Rodier, New challenges in polymer foaming: A review of extrusion processes assisted by supercritical carbon dioxide, Progress in Polymer Science 36(6) (2011) 749-766.

[53] Z. Luo, J. Shi, S.P. Beeby, Novel thick-foam ferroelectret with engineered voids for energy harvesting applications, Journal of Physics: Conference Series 773(1) (2016) 012030.

[54] C.B. Yan Zhang, Sylvain Deville, Ice-templated poly(vinylidence fluoride) ferroelectrets, ARXIV, 2018, p. 2018arXiv180909708Z.

[55] B. Wang, J. Zhong, Q. Zhong, N. Wu, X. Cheng, W. Li, K. Liu, L. Huang, B. Hu, J. Zhou, Sandwiched Composite Fluorocarbon Film for Flexible Electret Generator, Advanced Electronic Materials 2(4) (2016) 1500408.

[56] J. Shi, D. Zhu, S. Beeby, An investigation of PDMS structures for optimized ferroelectret performance, Journal of physics: conference series, IOP Publishing, 2014, p. 012104.

[57] J. Shi, Z. Luo, D. Zhu, S.P. Beeby, PDMS/PVA composite ferroelectret for improved energy harvesting performance, Journal of Physics: Conference Series 773(1) (2016) 012051.

[58] A. Kachroudi, S. Basrour, L. Rufer, F. Jomni, Piezoelectric cellular micro-structured PDMS material for micro-sensors and energy harvesting, Journal of Physics: Conference Series, IOP Publishing, 2015, p. 012040. 
[59] K. Achraf, B. Skandar, R. Libor, S. Alain, J. Fathi, Micro-structured PDMS piezoelectric enhancement through charging conditions, Smart Materials and Structures 25(10) (2016) 105027.

[60] A. Kachroudi, S. Basrour, L. Rufer, A. Sylvestre, F. Jomni, Dielectric properties modelling of cellular structures with PDMS for micro-sensor applications, Smart Materials and Structures 24(12) (2015) 125013.

[61] S.K. Ghosh, T.K. Sinha, B. Mahanty, S. Jana, D. Mandal, Porous polymer composite membrane based nanogenerator: A realization of self-powered wireless green energy source for smart electronics applications, Journal of Applied Physics 120(17) (2016) 174501.

[62] S.K. Ghosh, T.K. Sinha, B. Mahanty, D. Mandal, Self-poled Efficient Flexible Ferroelectretic" Nanogenerator: A New Class of Piezoelectric Energy Harvester, Energy Technology 3(12) (2015) 1190-1197.

[63] B. Mahanty, S.K. Ghosh, S. Garain, D. Mandal, An effective flexible wireless energy harvester/sensor based on porous electret piezoelectric polymer, Materials Chemistry and Physics 186 (2017) 327-332.

[64] C. Xu, L. Zhang, Y. Xu, Z. Yin, Q. Chen, S. Ma, H. Zhang, R. Huang, C. Zhang, L. Jin, Filling the holes in piezopolymers with a solid electrolyte: a new paradigm of polingfree dynamic electrets for energy harvesting, Journal of Materials Chemistry A 5(1) (2017) 189-200.

[65] A. Mellinger, M. Wegener, W. Wirges, R.R. Mallepally, R. Gerhard-Multhaupt, Thermal and Temporal Stability of Ferroelectret Films Made from Cellular Polypropylene/Air Composites, Ferroelectrics 331(1) (2006) 189-199.

[66] P. Adhikary, S. Garain, D. Mandal, The co-operative performance of a hydrated salt assisted sponge like P (VDF-HFP) piezoelectric generator: an effective piezoelectric based energy harvester, Physical Chemistry Chemical Physics 17(11) (2015) 72757281.

[67] S.K. Ghosh, A. Biswas, S. Sen, C. Das, K. Henkel, D. Schmeisser, D. Mandal, Yb3+ assisted self-polarized PVDF based ferroelectretic nanogenerator: A facile strategy of highly efficient mechanical energy harvester fabrication, Nano Energy 30 (2016) 621629. 
[68] X. Ma, X. Zhang, Low cost electrostatic vibration energy harvesters based on negatively-charged polypropylene cellular films with a folded structure, Smart Materials and Structures 26(8) (2017) 085001.

[69] Z. Luo, D. Zhu, J. Shi, S. Beeby, C. Zhang, P. Proynov, B. Stark, Energy harvesting study on single and multilayer ferroelectret foams under compressive force, IEEE Transactions on Dielectrics and Electrical Insulation 22(3) (2015) 1360-1368.

[70] G.M. Sessler, J. Hillenbrand, Electromechanical response of cellular electret films, Applied Physics Letters 75(21) (1999) 3405-3407.

[71] G. Sessler, P. Pondrom, X. Zhang, Stacked and folded piezoelectrets for vibrationbased energy harvesting, Phase Transitions 89(7-8) (2016) 667-677.

[72] X. Zhang, L. Wu, G.M. Sessler, Energy scavenging from vibration with two-layer laminated fluoroethylenepropylene piezoelectret films, Applications of Ferroelectric, International Symposium on Integrated Functionalities and Piezoelectric Force Microscopy Workshop (ISAF/ISIF/PFM), 2015 Joint IEEE International Symposium on the, IEEE, 2015, pp. 24-27.

[73] X. Zhang, P. Pondrom, L. Wu, G. Sessler, Vibration-based energy harvesting with piezoelectrets having high d 31 activity, Applied Physics Letters 108(19) (2016) 193903.

[74] Z. Luo, J. Shi, S. Beeby, Novel thick-foam ferroelectret with engineered voids for energy harvesting applications, Journal of Physics: Conference Series, IOP Publishing, 2016, p. 012030.

[75] R. Kressmann, Linear and nonlinear piezoelectric response of charged cellular polypropylene, Journal of Applied Physics 90(7) (2001) 3489-3496.

[76] S.R. Anton, K.M. Farinholt, An evaluation on low-level vibration energy harvesting using piezoelectret foam, Active and Passive Smart Structures and Integrated Systems 2012, International Society for Optics and Photonics, 2012, p. 83410G.

[77] S. Anton, K. Farinholt, A. Erturk, Piezoelectret foam-based vibration energy harvesting, Journal of Intelligent Material Systems and Structures 25(14) (2014) 16811692.

[78] C.A. Ray, S.R. Anton, Evaluation of piezoelectret foam in a multilayer stack configuration for low-level vibration energy harvesting applications, Active and 
Passive Smart Structures and Integrated Systems 2015, International Society for Optics and Photonics, 2015, p. 943111.

[79] A. Kachroudi, S. Basrour, L. Rufer, F. Jomni, Piezoelectric characterizations of piezo-electret PDMS material for energy harvesting, 6èmes Journées Nationales sur la Récupération et le Stockage d'Energie (JNRSE'16), 2016.

[80] P. Pondrom, J. Hillenbrand, G. Sessler, J. Bös, T. Melz, Vibration-based energy harvesting with stacked piezoelectrets, Applied Physics Letters 104(17) (2014) 172901.

[81] P. Pondrom, G. Sessler, J. Bös, T. Melz, Compact electret energy harvester with high power output, Applied Physics Letters 109(5) (2016) 053906.

[82] D.P. Almond, C.R. Bowen, An Explanation of the Photoinduced Giant Dielectric Constant of Lead Halide Perovskite Solar Cells, The Journal of Physical Chemistry Letters 6(9) (2015) 1736-1740.

[83] Y. Wu, X. Zhao, F. Li, Z. Fan, Evaluation of Mixing Rules for Dielectric Constants of Composite Dielectrics by MC-FEM Calculation on 3D Cubic Lattice, Journal of Electroceramics 11(3) (2003) 227-239.

[84] B. Notario, J. Pinto, M.A. Rodriguez-Perez, Nanoporous polymeric materials: A new class of materials with enhanced properties, Progress in Materials Science 78-79 (2016) 93-139.

[85] H.V. M. Paajanen, and J. Lekkala, Proceedings of the Tenth International Symposium on Electrets, Delphi, Greece, 22-24 September, 1999 (IEEE Service Center, Piscataway, NJ, 1999), pp. 735-738.

[86] S. Kärki, J. Lekkala, A lumped-parameter transducer model for piezoelectric and ferroelectret polymers, Measurement 45(3) (2012) 453-458.

[87] C. Ennawaoui, A. Hajjaji, A. Azim, Y. Boughaleb, Theoretical modeling of power harvested by piezo-cellular polymers, Molecular Crystals and Liquid Crystals 628(1) (2016) 49-54.

[88] J. Hillenbrand, P. Pondrom, G. Sessler, Electret transducer for vibration-based energy harvesting, Applied Physics Letters 106(18) (2015) 183902.

[89] J. Shi, D. Zhu, Z. Cao, S. Beeby, Optimization of a PDMS structure for energy harvesting under compressive forces, Journal of Physics: Conference Series, IOP Publishing, 2015, p. 012041. 
[90] Z. Luo, D. Zhu, S. Beeby, An electromechanical model of ferroelectret for energy harvesting, Smart Materials and Structures 25(4) (2016) 045010.

[91] P. Pondrom, J. Hillenbrand, G.M. Sessler, J. Bös, T. Melz, Vibration-based energy harvesting with stacked piezoelectrets, Applied Physics Letters 104(17) (2014) 172901.

[92] P. Pondrom, J. Hillenbrand, G.M. Sessler, J. Bos, T. Melz, Energy harvesting with single-layer and stacked piezoelectret films, IEEE Transactions on Dielectrics and Electrical Insulation 22(3) (2015) 1470-1476.

[93] C.A. Ray, S.R. Anton, Multilayer piezoelectret foam stack for vibration energy harvesting, Journal of Intelligent Material Systems and Structures 28(3) (2017) 408420.

[94] D. Gross, B.-X. Xu, Micromechanical Modelling of Cellular Ferroelectrets by Using Internal Variables, Procedia IUTAM 12 (2015) 62-72.

[95] W. Li, D. Torres, T. Wang, C. Wang, N. Sepúlveda, Flexible and biocompatible polypropylene ferroelectret nanogenerator (FENG): on the path toward wearable devices powered by human motion, Nano energy 30 (2016) 649-657.

[96] Y. Wu, Y. Hu, Z. Huang, C. Lee, F. Wang, Electret-material enhanced triboelectric energy harvesting from air flow for self-powered wireless temperature sensor network, Sensors and Actuators A: Physical 271 (2018) 364-372.

[97] Z. Luo, D. Zhu, S. Beeby, Multilayer ferroelectret-based energy harvesting insole, Journal of Physics: Conference Series, IOP Publishing, 2015, p. 012118.

[98] L.W. Xiaoqing Zhang, Gerhard M. Sessler, Energy scavenging from vibration with two-layer

laminated fluoroethylenepropylene piezoelectret

films, IEEE International Symposium on Applications of Ferroelectrics, Singapore, 2015, pp. 24-27.

[99] J. Strobel, S.J. Rupitsch, R. Lerch, Ferroelectret sensor array for characterization of cavitation effects in ultrasonic cleaning, 2009 IEEE International Ultrasonics Symposium, 2009, pp. 1-4.

[100] B. Wang, C. Liu, Y. Xiao, J. Zhong, W. Li, Y. Cheng, B. Hu, L. Huang, J. Zhou, Ultrasensitive cellular fluorocarbon piezoelectret pressure sensor for self-powered human physiological monitoring, Nano Energy 32(Supplement C) (2017) 42-49. 
